\title{
KINETIC THEORY FOR NEURONAL NETWORK DYNAMICS*
}

\author{
DAVID $\mathrm{CAI}^{\dagger}$, LOUIS TAO ${ }^{\ddagger}$, AADITYA V. RANGAN ${ }^{\S}$, AND DAVID W. MCLAUGHLIN $₫$
}

\begin{abstract}
We present a detailed theoretical framework for statistical descriptions of neuronal networks and derive $(1+1)$-dimensional kinetic equations, without introducing any new parameters, directly from conductance-based integrate-and-fire neuronal networks. We describe the details of derivation of our kinetic equation, proceeding from the simplest case of one excitatory neuron, to coupled networks of purely excitatory neurons, to coupled networks consisting of both excitatory and inhibitory neurons. The dimension reduction in our theory is achieved via novel moment closures. We also describe the limiting forms of our kinetic theory in various limits, such as the limit of mean-driven dynamics and the limit of infinitely fast conductances. We establish accuracy of our kinetic theory by comparing its prediction with the full simulations of the original point-neuron networks. We emphasize that our kinetic theory is dynamically accurate, i.e., it captures very well the instantaneous statistical properties of neuronal networks under time-inhomogeneous inputs.
\end{abstract}

Key words. Visual cortex, coarse-grain, fluctuation, diffusion, correlation.

AMS subject classifications. 82C31, 92B99, 92C20

\section{Introduction}

Mathematically, the vast hierarchy of the multiple spatial and temporal scales in the cortical dynamics presents a significant challenge to computational neuroscience. While we may devise ever more efficient numerical methods for simulations of dynamics of large-scale neuronal networks $[1,2,3,4,5]$, basic computational constraints will eventually limit our simulation power. In order to "scale-up" computational models to sufficiently large regions of the cortex to capture interesting cortical processing (such as optical illusions related to "contour completion"), and to gain qualitative understanding of the cortical mechanisms underlying this level of cortical processing, a major theoretical issue is how to derive effective dynamics under a reduced representation of large-scale neuronal networks. Therefore, a theoretical challenge is to develop efficient and effective representations for simulating and understanding the dynamics of larger, multi-layered networks. As suggested, for example, by the laminar structure of cat's or monkey's primary visual cortex, in which many cellular properties such as orientation preference are arranged in regular patterns or maps across the cortex $[6,7,8,9,10]$, some neuronal sub-populations may be effectively represented by coarse-grained substitutes. Thus, we may partition the two-dimensional cortical layers into coarse-grained patches, each sufficiently large to contain many neurons, yet sufficiently small that these regular response properties of the individual neurons within each patch are approximately the same for each neuron in the patch. These regular response properties are then treated as constants throughout each coarse-grained patch.

${ }^{*}$ Received: October 12, 2005; accepted (in revised version): December 25, 2005. Communicated by Shi Jin.

${ }^{\dagger}$ Courant Institute of Mathematical Sciences, New York University, New York, NY 10012, USA (cai@cims.nyu.edu).

${ }^{\ddagger}$ Department of Mathematical Sciences, New Jersey Institute of Technology, Newark, NJ 07102, USA (tao@njit.edu).

$\S$ Courant Institute of Mathematical Sciences, New York University, New York, NY 10012, USA (rangan@cims.nyu.edu).

${ }^{\top}$ Courant Institute of Mathematical Sciences, New York University, New York, NY 10012, USA (dmac@cims.nyu.edu). 
Furthermore, there is another important issue that needs to be addressed in coarse-grained formalisms, i.e., how to capture fluctuation-dominated spiking processes mathematically, because neuronal networks, whether real cortical networks $[11,12]$ or computer models $[13,14,4]$, frequently operate in a regime in which spiking is caused by irregular temporal fluctuations of the membrane potential. In this cortical state, the mean membrane potential (say, obtained by averaging locally in time, or by trial averages, i.e., averaging over many voltage traces under the same stimulus condition), does not reach firing threshold, but, in each instance, fluctuations in the membrane potential do reach spiking threshold. We note in passing that, since a purely excitatory network can still operate in a fluctuation-driven regime, this notion of fluctuation-dominated dynamics is distinct from the notion of fluctuation near threshold in the so-called balanced network that uses near cancellation of excitatory and inhibitory currents $[15,16]$.

Previously, we briefly reported our results [17] of the development of an effective representation. Starting with large-scale model networks of point neurons, which are sufficiently detailed for modeling neuronal computation of large systems, we tiled the model cortex with coarse-grained patches. We then derived an effective dynamics to capture the statistical behavior of the many neurons within each coarse-grained patch in their interaction with other coarse-grained patches. As mentioned in Ref. [17], our kinetic theory of a purely excitatory network can be extended to realistic networks with excitatory and inhibitory populations of neurons. We have used this extended kinetic theory to study rich dynamic phenomena within these networks, including transitions to bistability and hysteresis, even in the presence of large fluctuations. We have also used these representations to study simplified models of orientation selectivity to suggest the roles of large fluctuations and cortico-cortical excitation in the orientation tuning of complex cells $[17,18]$. This kinetic theory approach has been shown to be rather powerful, allowing for both computational scale-up and structural insight into the mechanisms of cortical networks $[17,19]$.

In this paper, we present the detailed theoretical framework for capturing these coarse-grained dynamics that can be fluctuation-dominated. (For earlier probabilistic representations, upon which our coarse-grained theory is based, see, e.g., $[20,21,22,23,24,25,26,27,28,29,30,31,32,33,14])$. Starting with networks of conductance-based integrate-and-fire (I\&F) neurons, we derive a full kinetic description directly, without introduction of any new parameters. We present details of how to extend our novel moment closure to construct kinetic theories for networks with both excitatory and inhibitory neurons. In the limit of infinitely fast conductances, these kinetic equations can be further reduced to one-dimensional Fokker-Planck equations. As the number of point neurons $N$ tends to infinity, we show that our kinetic theory reduces further to the classical firing rate representation $[34,23,13]$. Through this sequence of mathematical reductions, we can gain analytical insights into the behavior of various dynamic regimes of neuronal networks. As pointed out in Ref. [17], at moderate and even small $N$, this kinetic theory captures the effects of large fluctuations, with numerical efficiency and surprising accuracy. Here, we further establish dynamical accuracy of our kinetic theory by benchmarking its predictions against the full simulations of the point neuron network under time-inhomogeneous inputs. Although the derivation of our kinetic theory assumes that the number of neurons in a coarse-grained patch is large, the numerical verification of this asymptotic shows that the number $N$ can become as small as $\mathcal{O}(10)$, with accuracy retained [17]. As expected $[35,36,33,17]$, the kinetic representation is far more efficient computationally 
than the full I\&F network. The savings are two-fold: (i) For a given I\&F network, the probability density representation eliminates the need for simulating to very long times or for simulating many ensembles so as to reduce the statistical error in computing firing rates; (ii) The reduction of the dimension, achieved by our closure, for a probability density description provides truly significant computational savings. For example, to achieve a firing rate computation within $1 \%$ accuracy for a network of 100 neurons, a reduction in computation time of $4-6$ orders of magnitude can be easily obtained $[17,37]$.

The paper is organized as follows: In Sec. 2, we briefly describe the dynamics of conductance-based integrate-and-fire neuronal networks; In Sec. 3, for the dynamics of a single I\&F neuron, with an infinitely fast conductance, driven by a Poisson input spike train, we derive an exact kinetic equation and study its properties under a diffusion approximation; In Sec. 4, using a conditional variance closure, we describe in detail the derivation of kinetic equations for all-to-all coupled networks of purely excitatory neurons; In Sec. 5, we extend this kinetic theory to coupled networks consisting of both excitatory and inhibitory neurons; Finally, in Sec. 6 we indicate how to generalize our kinetic theory to multiple interacting coarse-grained spatial patches.

\section{Integrate-and-fire neuronal networks}

For convenience of description, we briefly review conductance-based integrateand-fire (I\&F) neuronal networks [38]. We consider the following all-to-all coupled network of $N_{E}$ excitatory neurons and $N_{I}$ inhibitory neurons, with its I\&F dynamics of $i^{\text {th }}$ neuron's membrane potential, $V_{i}^{\lambda}$, of $\lambda$-type governed by

$$
\begin{aligned}
\tau \frac{d V_{i}^{\lambda}}{d t} & =-\left(V_{i}^{\lambda}-\varepsilon_{r}\right)-G_{i}^{\lambda E}(t)\left(V_{i}^{\lambda}-\varepsilon_{E}\right)-G_{i}^{\lambda I}(t)\left(V_{i}^{\lambda}-\varepsilon_{I}\right), \\
\sigma_{E} \frac{d G_{i}^{\lambda E}}{d t} & =-G_{i}^{\lambda E}+\sum_{\mu} f_{E} \delta\left(t-t_{\mu}^{i}\right)+\frac{S_{\lambda E}}{N_{E}} \sum_{j \in \mathcal{P}_{E}} \sum_{\mu} p_{j \mu}^{E} \delta\left(t-t_{j \mu}^{E}\right), \\
\sigma_{I} \frac{d G_{i}^{\lambda I}}{d t} & =-G_{i}^{\lambda I}+\sum_{\mu} f_{I} \delta\left(t-T_{\mu}^{i}\right)+\frac{S_{\lambda I}}{N_{I}} \sum_{j \in \mathcal{P}_{I}} \sum_{\mu} p_{j \mu}^{I} \delta\left(t-t_{j \mu}^{I}\right),
\end{aligned}
$$

with $\lambda=E, I$ labeling excitation and inhibition. Whenever $V_{i}^{\lambda}(t)$ crosses the firing (spiking) threshold $V_{T}$, a spike is recorded with the spike-time $t_{s p}$ such that $V_{i}^{\lambda}\left(t_{s p}^{-}\right)=$ $V_{T}$ and $V_{i}^{\lambda}\left(t_{s p}^{+}\right)=\varepsilon_{r}$, where $\varepsilon_{r}$ is the reset voltage. Here, $G_{i}^{\lambda E}(t)$ and $G_{i}^{\lambda I}(t)$ are the excitatory and inhibitory conductances of the $i$ th neuron of $\lambda$-type, with corresponding reversal potentials $\varepsilon_{E}$ and $\varepsilon_{I}$, respectively. Note that, from physiology, we have $\varepsilon_{I} \leq$ $\varepsilon_{r}<V_{T}<\varepsilon_{E}=0 \mathrm{mV}$. (Typical values are $\varepsilon_{I}=-80 \mathrm{mV}, \varepsilon_{r}=-65 \mathrm{mV}, V_{T}=-55 \mathrm{mV}$, and $\varepsilon_{E}=0 \mathrm{mV}$.) $\tau$ is the membrane time constant of membrane potential, whereas $\sigma_{E}$ and $\sigma_{I}$ are decay time constants of excitatory and inhibitory conductances, respectively. For external inputs, we assume that, the $i$ th neuron of the excitatory (inhibitory) population receives external spikes, $\left\{t_{\mu}^{i}\right\}\left(\left\{T_{\mu}^{i}\right\}\right)$, each of which is an independent realization of the Poisson process with rate $\nu_{0 E}(t)\left(\nu_{0 I}(t)\right) . t_{j \mu}^{E} \quad\left(t_{j \mu}^{I}\right)$ is the spiketime of the $\mu$ th spike of the $j$ th excitatory (inhibitory) neuron. Note that, under Poisson external inputs, spiking events of a neuron in the network, in general, are not Poisson, i.e., for a fixed $j,\left\{t_{j \mu}^{E}\right\}$ and $\left\{t_{j \mu}^{I}\right\}$ are not Poisson, in general. The parameter $f_{\lambda}$ determines the size of conductance increase associated with a spike from the external input. The parameters $S_{\lambda E}\left(S_{\lambda I}\right)$ describe the strength of network 
couplings from the excitatory (inhibitory) population to the $\lambda$-population of neurons. $\mathcal{P}_{E}\left(\mathcal{P}_{I}\right)$ denotes the set of excitatory (inhibitory) neurons. The factor $1 / N_{E, I}$ ensures that there is a well-defined network coupling in the limit of $N_{E, I} \rightarrow \infty \cdot p_{j \mu}^{E, I}$ describes the probability of synaptic release upon arrival of each spike. The synaptic release is modeled as a Bernoulli process, with release probability equal to $p$, i.e., $p_{j \mu}^{E, I}=1$ with probability $p ; 0$, otherwise.

We note that, in our coarse-grained procedure as discussed in Introduction, the network described by Eqs. (2.1) can be viewed as a network in a coarse-grained patch, as its architectural properties are approximated as uniform over the patch. This is the motivation behind our consideration of the coupled network with these particular all-to-all couplings. As described above, the external input for Eqs. (2.1) is assumed to be Poisson. This can be intuitively justified along the same spirit of coarse-graining: There are many spike-trains from many neurons in other patches. The spike-train to one neuron in the network described by Eqs. (2.1) is a spike process summing over all incoming spike-trains. Therefore, under certain conditions, this input process can be naturally approximated by Poisson [39]. Finally, we comment that the process of synaptic release as described in the end of the last paragraph is not crucial for our coarse-grained dynamics as far as the closure argument is concerned. However, we mention this model of synaptic release here for the purpose of comparison between the kinetic theory we derived below and the full, original I\&F neuronal dynamics. The synaptic release as modeled above can often induce the system to operate in an asynchronous firing regime, in contrast to a regime, say, with a spiketo-spike synchrony that can appear in a more regularly structured network. It is to asynchronous firing regimes that the kinetic theory can be very successfully applied with high accuracy.

\section{Probabilistic description of a single neuron}

3.1. Evolution of probability density $\rho$. First, we illustrate how to turn the description of the trajectory of a neuron into a probabilistic description of its dynamics by way of deriving an equation governing the evolution of a probability density function (pdf). As motivation for the realistic extensions which follow, we consider the simplest case, in which there is only one excitatory neuron with its conductance time scale being infinitely fast, i.e., $\sigma \equiv \sigma_{E} \rightarrow 0$. Between any two firings of this I\&F neuron, the dynamics of the integrate-and-fire neuron now is governed by

$$
\frac{d}{d t} V=-\frac{V-\varepsilon_{r}}{\tau}-\frac{f}{\tau}\left[\sum_{k} \delta\left(t-t_{k}\right)\right]\left(V-\varepsilon_{E}\right),
$$

where $V(t)$ is the membrane potential of the neuron and $\left\{t_{k}\right\}$ is a Poisson process with rate $\nu(t)$. Note that the Dirac- $\delta$ function in Eq. (3.1) comes from the conductance time-course from Eq. (2.1b) in the $\sigma \rightarrow 0$ limit, $\lim _{\sigma \rightarrow 0} \frac{f}{\sigma} e^{-\frac{t}{\sigma}} \theta(t)=f \delta(t)$, where $\theta(t)$ is the Heaviside function.

3.1.1. Solutions for trajectories. Note that, the solution of Eq. (3.1) should be interpreted as the $\sigma \rightarrow 0$ limit of the solution of the following I\&F neuronal dynamics:

$$
\begin{aligned}
\tau \frac{d V}{d t} & =-\left(V-\varepsilon_{r}\right)-G(t)\left(V-\varepsilon_{E}\right), \\
\sigma \frac{d G}{d t} & =-G+f \sum_{k} \delta\left(t-t_{k}\right),
\end{aligned}
$$


in which the decay time constant $\sigma$ of the conductance is finite. In particular, for a single input spike arriving at time $t=0$, it is easy to show the solution $V(t)$ thus obtained in the limit of $\sigma \rightarrow 0$ has a jump between $V\left(0^{+}\right)$and $V\left(0^{-}\right)$, which satisfies

$$
V\left(0^{+}\right)-V\left(0^{-}\right)=\left(\varepsilon_{E}-V\left(0^{-}\right)\right) \Gamma
$$

where $\Gamma \equiv 1-\exp \left(-\frac{f}{\tau}\right)$. (See Appendix A for a simple formal way of obtaining the result (Eq. (3.3))). The voltage of the neuron, after the jump induced by the input spike-time $t_{k}$, follows the leakage dynamics $\dot{V}=-\left(V-\varepsilon_{r}\right) / \tau$ (until the next external input spike arrives) and is simply given by

$$
V(t)=\left(V\left(t_{k}^{+}\right)-\varepsilon_{r}\right) \exp \left(-\frac{t-t_{k}}{\tau}\right)+\varepsilon_{r}
$$

for $V\left(t_{k}^{+}\right)<V_{T}$. If, upon receiving the input spike $t_{k}$, the jump (3.3) indicates that $V\left(t_{k}^{+}\right) \geq V_{T}$, then $V\left(t_{k}^{+}\right)$is reset to $\varepsilon_{r}$, i.e., $V\left(t_{k}^{+}\right)=\varepsilon_{r}$. Therefore, for a given realization of Poisson input spikes $\left\{t_{k}\right\}$, we can easily piece together the whole solution of $V(t)$ following I\&F dynamics.

3.1.2. Kinetic equation. Now we change the point of view from that of following an individual trajectory to a statistical one. First, we construct an ensemble of trajectories that correspond to independent realizations of Poisson input spike trains. Then, we ask what is the probability of finding a neuron in this ensemble which, at time $t$, has membrane potential within the interval $(v, v+d v)$. Clearly, the corresponding probability density is

$$
\rho(v, t)=\mathbb{E}[\delta(v-V(t))]
$$

where $\mathbb{E}(\cdot)$ is the expectation with respect to all independent realizations of the input Poisson spike process with rate $\nu(t)$ for given initial data. (Note that we can further average over an ensemble of initial conditions $V(0)$ if necessary.)

We invoke the property of conditional expectation, in our setting, that is,

$$
\mathbb{E}[\delta(v-V(t+\Delta t))]=\mathbb{E}[\mathbb{E}[\delta(v-V(t+\Delta t)) \mid V(t)]]
$$

i.e., an average over all trajectories at time $t+\Delta t$ can be achieved by, (i) first, in the interval $[t, t+\Delta t]$, averaging over all trajectories conditioned on a fixed $V(t)$ at time $t$ (i.e., over all those trajectories starting from $V(t)$ at time $t$ ), (ii) then, in the interval $[0, t]$, averaging over all possible trajectories terminating at $V(t)$ at time $t$, for each possible value of $V(t)$. For a neuron whose voltage is $V(t)$ at time $t$, there are two possibilities in the interval $(t, t+\Delta t)$ - either receiving an input spike or not. For a Poisson input, the probability of receiving an input spike is $\nu \Delta t$ for a small interval $\Delta t$ and the probability of receiving no spike is $(1-\nu \Delta t)$. If the neuron receives a spike, the voltage will have a jump described by Eq. (3.3). Otherwise, the neuron will follow the streaming dynamics of relaxation $\dot{V}=-\left(V-\varepsilon_{r}\right) / \tau$. Therefore, up to $\mathcal{O}(\Delta t)$, we have the conditional expectation

$$
\begin{aligned}
\mathbb{E}[\delta(v-V(t+\Delta t)) \mid V(t)]= & \nu \Delta t \delta\left(v-\left[V(t)+\left(\varepsilon_{E}-V(t)\right) \Gamma\right]\right) \\
& +(1-\nu \Delta t) \delta\left(v-\left[V(t)+\left(-\frac{V(t)-\varepsilon_{r}}{\tau}\right) \Delta t\right]\right),
\end{aligned}
$$


where the first term describes the jump and its associated probability whereas the second term describes the streaming of membrane potential and its associated probability. Note that the second term in Eq. (3.6) can be expanded up to $\mathcal{O}(\Delta t)$ as

$$
\begin{aligned}
\delta\left(v-\left[V(t)+\left(-\frac{V(t)-\varepsilon_{r}}{\tau}\right) \Delta t\right]\right) & =\delta(v-V(t))+\left(\frac{\partial}{\partial v} \delta(v-V(t))\right)\left[\frac{V(t)-\varepsilon_{r}}{\tau}\right] \Delta t \\
& =\delta(v-V(t))+\frac{\partial}{\partial v}\left(\delta(v-V(t))\left[\frac{V(t)-\varepsilon_{r}}{\tau}\right]\right) \Delta t \\
& =\delta(v-V(t))+\frac{\partial}{\partial v}\left(\delta(v-V(t))\left[\frac{v-\varepsilon_{r}}{\tau}\right]\right) \Delta t,
\end{aligned}
$$

by using $a \delta(x-a)=x \delta(x-a)$ inside the large parentheses. For the first term in Eq. (3.6), within $\Delta t$, beside the jump, there is still a streaming of $V$ under the relaxation leakage dynamics. However, its contribution is $O(\Delta t)$ in the argument of the $\delta$-function, combining with $\nu \Delta t$ to yield a higher order $O\left(\Delta t^{2}\right)$-term. As a consequence, this term does not need to take into account the streaming contribution in the interval $[t, t+\Delta t]$. Eqs. (3.4) and (3.5) yield

$$
\begin{aligned}
& \rho(v, t+\Delta t)=\mathbb{E}[\delta(v-V(t+\Delta t))] \\
& =\nu \Delta t \mathbb{E}\left[\delta\left(v-V(t)-\Gamma\left(\varepsilon_{E}-V(t)\right)\right)\right] \\
& \quad+(1-\nu \Delta t) \mathbb{E}\left[\delta(v-V(t))+\frac{\partial}{\partial v}\left[\delta(v-V(t))\left(\frac{v-\varepsilon_{r}}{\tau}\right)\right] \Delta t\right] \\
& =\frac{\nu \Delta t}{1-\Gamma} \rho\left(v-\frac{\Gamma}{1-\Gamma}\left(\varepsilon_{E}-v\right)\right)+(1-\nu \Delta t)\left(\rho(v, t)+\frac{\partial}{\partial v}\left[\rho(v, t)\left(\frac{v-\varepsilon_{r}}{\tau}\right)\right] \Delta t\right)
\end{aligned}
$$

where use is made of the fact that, by definition,

$$
\begin{aligned}
\rho\left(v-\frac{\Gamma}{1-\Gamma}\left(\varepsilon_{E}-v\right)\right) & =\mathbb{E}\left[\delta\left(v-\frac{\Gamma}{1-\Gamma}\left(\varepsilon_{E}-v\right)-V(t)\right)\right] \\
& =(1-\Gamma) \mathbb{E}\left[\delta\left(v-V(t)-\Gamma\left(\varepsilon_{E}-V(t)\right)\right)\right]
\end{aligned}
$$

Taking the limit $\Delta t \rightarrow 0$, we obtain the equation governing the evolution of the pdf $\rho(v, t)$ :

$$
\partial_{t} \rho(v, t)=\partial_{v}\left[\left(\frac{v-\varepsilon_{r}}{\tau}\right) \rho(v, t)\right]+\nu(t)\left[\frac{1}{1-\Gamma} \rho\left(v-\frac{\Gamma}{1-\Gamma}\left(\varepsilon_{E}-v\right), t\right)-\rho(v, t)\right]
$$

for $v \in\left[\varepsilon_{r}, V_{T}\right)$. Eq. (3.7) constitutes the kinetic equation for the single neuron dynamics. Note that Eq. (3.7) is an exact kinetic equation for the I\&F dynamics (3.1) without any approximations.

Eq. (3.7) can be cast into a form of the conservation of probability

$$
\partial_{t} \rho(v, t)+\partial_{v} J(v, t)=0
$$

by defining the probability flux $J(v, t)=J_{r}(v, t)+J_{\nu}(v, t)$, where

$$
J_{r}(v, t) \equiv-\left(\frac{v-\varepsilon_{r}}{\tau}\right) \rho(v, t),
$$


is a streaming flux due to the relaxation dynamics, and

$$
J_{\nu}(v, t) \equiv \nu(t) \int_{\varepsilon_{r}}^{v}\left[\rho(u, t)-\frac{1}{1-\Gamma} \rho\left(u-\frac{\Gamma}{1-\Gamma}\left(\varepsilon_{E}-u\right), t\right)\right] d u
$$

is an input-driven flux induced by the voltage jumps upon receiving input spikes. In general, the input-driven flux $J_{\nu}$ can be written as

$$
J_{\nu}(v, t)=\nu(t) \int_{\varepsilon_{r}}^{v} \rho(u, t) d u-\nu(t) \int_{\varepsilon_{r} /(1-\Gamma)}^{v /(1-\Gamma)} \rho(u, t) d u
$$

where, for notational simplicity, we have set $\varepsilon_{E}=0$, which is the physiologically realistic value for $\varepsilon_{E}$. Note that $0>\varepsilon_{r}>\varepsilon_{r} /(1-\Gamma)$, and $0>v>v /(1-\Gamma)$. Since $\rho(v)=0$ for $v$ outside $\left[\varepsilon_{r}, V_{T}\right]$, it is easy to see that

$$
J_{\nu}(v, t)=\nu(t) \int_{v /(1-\Gamma)}^{v} \rho(u, t) d u
$$

As conforming with intuition, Eq. (3.10) shows that the input-driven flux $J_{\nu}(v, t)$ is proportional to the input rate $\nu(t)$, and the input-driven flux crossing the voltage $v$ depends on $\rho$ only in a voltage layer below $v$, i.e., $(v /(1-\Gamma), v)$, because all neurons, in the ensemble, with voltages in the interval $(v /(1-\Gamma), v)$ will move across $v$ upon receiving an input spike (see Eq. (3.3) with $\varepsilon_{E}=0$ ).

BOUNDARY CONDITIONS: Note that, the neuron in this setting with an infinitely fast conductance can only fire by receiving a spike and it cannot stream up to cross the firing threshold. Since $V_{T}>\varepsilon_{r}$, we have, in general,

$$
\left.J_{r}(v, t)\right|_{v=V_{T}}=-\left(\frac{V_{T}-\varepsilon_{r}}{\tau}\right) \rho\left(V_{T}, t\right) \leq 0 .
$$

A negative streaming flux at $V=V_{T}$ is impossible since the neuron can never stream down via the relaxation leakage dynamics at $V_{T}$ - it can only reset once it arrives at the threshold. Therefore, $J_{r}\left(V_{T}, t\right) \equiv 0$. Hence,

$$
\rho\left(V_{T}, t\right) \equiv 0,
$$

which is a boundary condition for solving Eq. (3.7).

FIRING RATE: The neuronal dynamics is quantified by its firing rate in response to the external input with rate $\nu(t)$. The firing rate is the probability per unit time for the neuron moving across the threshold $V_{T}$, which is simply the probability flux across the threshold:

$$
m(t)=J\left(V_{T}, t\right)=J_{\nu}\left(V_{T}, t\right) .
$$

From Eq. (3.10), we obtain

$$
m(t)=\nu(t) \int_{V_{T} /(1-\Gamma)}^{V_{T}} \rho(u, t) d u .
$$

As conforming with intuition, Eq. (3.11) shows that, for the single neuron case, the firing rate $m(t)$ is proportional to the external input rate $\nu(t)$ and depends on $\rho(v, t)$ only within a thin layer near the threshold $V_{T}$, if jump sizes are small, i.e., $f$ is small and $V_{T} /(1-\Gamma) \approx V_{T}(1+f / \tau)$. 
3.2. Diffusion approximation. The exact kinetic equation (3.7) for the single I\&F neuronal dynamic (3.1) is a partial differential equation with a delay in $v$-space (i.e., $\partial_{t} \rho(v)$ at $v$ depends on $\rho$ at a finite distance away, that is, at $v-$ $\left.\frac{\Gamma}{1-\Gamma}\left(\varepsilon_{E}-v\right)\right)$. If $f$ is sufficiently small, we can derive a diffusion equation as an approximation to Eq. (3.7) to gain insight into the I\&F dynamics. Since $1 /(1-\Gamma) \approx$ $1+f / \tau+f^{2} /\left(2 \tau^{2}\right)$, the length of the integration interval in the integral in Eq. (3.10) is $\mathcal{O}(f)$. Using the trapezoidal rule to approximate the integral, it is easy to show that, to $\mathcal{O}\left(f^{2}\right)$, Eq. (3.10) can be written as

$$
J_{\nu}(v, t)=-\nu(t)\left[\left(\frac{f}{\tau}+\frac{f^{2}}{2 \tau^{2}}\right) \rho(v) v+\frac{f^{2}}{2 \tau^{2}} v^{2} \frac{\partial \rho}{\partial v}\right]+O\left(f^{3}\right)
$$

which, by substituting, together with Eq. (3.9), into Eq. (3.8), yields the FokkerPlanck equation:

$$
\partial_{t} \rho(v, t)=\partial_{v}\left\{\frac{1}{\tau}\left[v-\varepsilon_{r}+\nu(t)\left(f+\frac{f^{2}}{2 \tau}\right) v\right] \rho(v, t)+\frac{f^{2} \nu(t)}{2 \tau^{2}} v^{2} \partial_{v} \rho(v, t)\right\}
$$

for $v \in\left[\varepsilon_{r}, V_{T}\right)$. We note that we use $\varepsilon_{E}=0$ in the derivation of Eq. (3.12).

As is assumed, the input spike train is a Poisson point process. If the rate $\nu(t)$ is sufficiently high, intuitively, we can approximate the conductance $G(t)=$ $f\left[\sum_{k} \delta\left(t-t_{k}\right)\right]$ in Eq. (3.1) by $G(t)=\bar{G}(t)+\sigma_{G} \zeta$ with the mean $\bar{G}(t)=f \nu(t)$ and variance $\sigma_{G}^{2}=f^{2} \nu(t)$, where $\zeta$ is a Gaussian-white noise with

$$
\begin{aligned}
\langle\zeta(t)\rangle & =0 \\
\left\langle\zeta(t) \zeta\left(t^{\prime}\right)\right\rangle & =\delta\left(t-t^{\prime}\right) .
\end{aligned}
$$

Then, the governing equation of the I\&F neuron evolution (3.1) is replaced by the Langevin equation:

$$
\tau \frac{d V}{d t}=-\left(V-\varepsilon_{r}\right)-\left(V-\varepsilon_{E}\right)\left[f \nu(t)+\sigma_{G} \zeta\right] .
$$

It is important to point out that the diffusion approximation (Eq. (3.12)) is the corresponding Kolmogorov forward equation for the diffusion process (3.13) when interpreted according to the Stratonovich sense, i.e.,

$$
\tau d V=-\left[\left(V-\varepsilon_{r}\right)+f \nu(t)\left(V-\varepsilon_{E}\right)\right] d t-\sigma_{G}\left(V-\varepsilon_{E}\right) \circ d W
$$

where $W(t)$ is a Wiener process.

\section{All-to-all coupled excitatory neuronal networks}

Going beyond the probabilistic description of the dynamics of a single neuron, we now turn to a discussion of a coupled network consisting of $N$ all excitatory neurons with an exponential time-course for conductances, i.e., Eqs. (2.1) without any inhibitory neurons, $N_{I} \equiv 0$. The dynamics of an all-to-all coupled excitatory I\&F neuronal network is governed by

$$
\begin{aligned}
\tau \frac{d V_{i}}{d t} & =-\left(V_{i}-\varepsilon_{r}\right)-G_{i}(t)\left(V_{i}-\varepsilon_{E}\right), \\
\sigma \frac{d G_{i}}{d t} & =-G_{i}+f \sum_{\mu} \delta\left(t-t_{\mu}^{i}\right)+\frac{S}{N} \sum_{j} \sum_{\mu} p_{j \mu} \delta\left(t-t_{j \mu}\right),
\end{aligned}
$$


where $V_{i}$ is the membrane potential of the $i$ th neuron in the network and $\sigma$ is the decay time-scale of the excitatory conductance time-course. $S$ is the strength of coupling between neurons in the network. $p_{j \mu}$ describes the probability of synaptic release, which is modelled by a Bernoulli process, with release probability equal to $p$, i.e., $p_{j \mu}=1$ with probability $p$; 0 , otherwise. For each incoming external spike, the jump in the conductance of a neuron is $f / \sigma$, and, for each incoming spike from other neurons in the network, the jump in the conductance is $S / N \sigma$.

As mentioned before, for a fixed neuron $j$, the output spike statistics of $\left\{t_{j \mu}\right\}$ are, in general, not Poisson. However, the input to the $i$ th neuron is a spike train summed over output spike trains from many neurons in the network. If we assume that each neuron firing event has a very low rate and is statistically independent from each other, then the spike train obtained by summing over a large number of output spike trains of neurons in the network asymptotically tends to be a Poisson spike process [39]. Therefore, in our derivation, we will assume that the input spike train summed from all other neurons to the $i$ th neuron is Poisson with rate $p N m(t)$, where $m(t)$ is the population-averaged firing rate per neuron and $p N$ is an effective number of neurons that are coupled to neuron $i$.

To study the statistical behavior of the network, we again construct a statistical ensemble of identically structured neuronal networks that differ only in their input, each of which is an independent set of $N$ independent realizations of the Poisson input spike trains with the same rate $\nu_{0}(t)$. We are interested in what is the probability of finding a neuron whose voltage is in $(v, v+d v)$ and whose conductance is in $(g, g+d g)$ at time $t$. The corresponding probability density function is

$$
\rho(v, g, t)=\mathbb{E}\left[\frac{1}{N} \sum_{i=1}^{N} \delta\left(v-V_{i}(t)\right) \delta\left(g-G_{i}(t)\right)\right],
$$

where $\mathbb{E}(\cdot)$ is the expectation with respect to all possible sets of $N$ independent realizations of the input Poisson spike process with rate $\nu_{0}(t)$ for given initial data (again, we can further average over an ensemble of initial conditions $V(0)$ if necessary.) Denote $\rho=\rho(v, g, t)$, the governing equation for the probability density is (see Appendix $\mathrm{B}$ for a detailed derivation)

$$
\begin{aligned}
\partial_{t} \rho= & \partial_{v}\left\{\left[\left(\frac{v-\varepsilon_{r}}{\tau}\right)+g\left(\frac{v-\varepsilon_{E}}{\tau}\right)\right] \rho\right\}+\partial_{g}\left(\frac{g}{\sigma} \rho\right) \\
& +\nu_{0}(t)\left[\rho\left(v, g-\frac{f}{\sigma}, t\right)-\rho(v, g, t)\right] \\
& +p m(t) N\left[\rho\left(v, g-\frac{\bar{S}}{p N \sigma}, t\right)-\rho(v, g, t)\right],
\end{aligned}
$$

for $v \in\left[\varepsilon_{r}, V_{T}\right)$ and $g \in[0, \infty)$, where $\bar{S}=p S$. The first two terms in Eq. (4.2) describe the streaming (i.e., relaxation) dynamics of neurons without receiving any spikes and the second term in Eq. (4.2a) describes the streaming arising from a finite $\sigma$, and is absent in Eq. (3.7). The third and fourth terms in Eq. (4.2) describe the conductance jumps of the neurons upon receiving external input spikes and spikes from other neurons in the network, respectively.

Again, Eq. (4.2) is a (2+1)-dimensional partial differential equation with delays in $g$-space. Unlike Eq. (3.7), Eq. (4.2) is not exact since the summed input from other neurons in the network is only approximately Poisson, and thus term (4.2c) is valid 
only in an asymptotic sense. To further shed light on the dynamics of the coupled I\&F network, we invoke a small-jump approximation to derive a $(2+1)$-dimensional, nonlinear advection-diffusion equation below, and discuss how to reduce the dynamics in two dimensions $(v, g)$ to dynamics in only one dimension $v$.

4.1. Diffusion approximation. Note that the jump in conductance of a neuron, induced by a single spike from another neuron in the network, is $\bar{S} /(p N \sigma)$, whereas the jump, induced by a single spike from the external input, is $f / \sigma$. Assuming that these jumps are small, we can expand terms $(4.2 b)$ and (4.2c) via Taylor expansion to obtain

$$
\partial_{t} \rho=\partial_{v}\left\{\left[\left(\frac{v-\varepsilon_{r}}{\tau}\right)+g\left(\frac{v-\varepsilon_{E}}{\tau}\right)\right] \rho\right\}+\partial_{g}\left\{\left[\frac{1}{\sigma}(g-\bar{g}(t)) \rho+\frac{\sigma_{g}^{2}(t)}{\sigma} \partial_{g} \rho\right]\right\}
$$

with

$$
\begin{aligned}
\bar{g}(t) & \equiv f \nu_{0}(t)+\bar{S} m(t) \\
\sigma_{g}^{2}(t) & \equiv \frac{1}{2 \sigma}\left[f^{2} \nu_{0}(t)+\frac{\bar{S}^{2}}{p N} m(t)\right] .
\end{aligned}
$$

Eq. (4.3) can be cast in the form of the conservation of probability:

$$
\partial_{t} \rho+\partial_{v} J_{V}(g, v)+\partial_{g} J_{G}(g, v)=0, \quad \text { for } v \in\left[\varepsilon_{r}, V_{T}\right), \text { and } g \in[0, \infty),
$$

with the flux

$$
\begin{aligned}
& J_{V}(v, g)=-\left[\left(\frac{v-\varepsilon_{r}}{\tau}\right)+g\left(\frac{v-\varepsilon_{E}}{\tau}\right)\right] \rho(v, g), \\
& J_{G}(v, g)=-\left[\frac{1}{\sigma}(g-\bar{g}(t)) \rho(v, g)+\frac{\sigma_{g}^{2}(t)}{\sigma} \partial_{g} \rho(v, g)\right],
\end{aligned}
$$

where the flux $J_{V}(v, g)$ and $J_{G}(v, g)$ are the flux along the $v$-direction and the $g$ direction, respectively.

Now we discuss boundary conditions. As described in Section 2, the reset dynamics of our I\&F neurons are instantaneous, i.e., once a neuron's voltage crosses the threshold $V_{T}$, the voltage resets immediately without any refractory period (i.e., without any delay) and the conductance stays with the same value upon voltage reset. Hence,

$$
J_{V}\left(V_{T}, g\right)=J_{V}\left(\varepsilon_{r}, g\right), \quad \text { for } \forall g \in[0, \infty) .
$$

Boundary condition (4.6) simply expresses the fact that the neurons that just fired all enter through the reset voltage. Furthermore, since there are no neurons whose conductance will go below zero or go to infinity, the $g$-flux vanishes at the boundary $g=0$ and $g=\infty$, i.e.,

$$
J_{G}(v, g=0)=0, \quad J_{G}(v, g=\infty)=0, \quad \text { for } \forall v \in\left[\varepsilon_{r}, V_{T}\right) .
$$

Eqs. (4.6) and (4.7) constitute boundary conditions for Eq. (4.3).

One of the most important statistical characterizations of neuronal networks is the firing rate, which is often measured in physiological experiments to describe neuronal 
network properties. Here, the dynamics of the network (4.1) are described by the population-averaged firing rate per neuron as determined by the total probability flux across the threshold regardless of the values of conductance, i.e.,

$$
\begin{aligned}
m(t) & =\int_{0}^{\infty} J_{V}\left(V_{T}, g, t\right) d g \\
& =-\int_{0}^{\infty}\left[\left(\frac{v-\varepsilon_{r}}{\tau}\right)+g\left(\frac{v-\varepsilon_{E}}{\tau}\right)\right] \rho\left(V_{T}, g, t\right) d g .
\end{aligned}
$$

Once the solution $\rho(v, g, t)$ is known, we can determine the firing rate via Eq. (4.8). However, Eq. (4.3) is specified with the parameters $\bar{g}(t)$ and $\sigma_{g}^{2}(t)$, which are functions of $m(t)$ (see Eqs (4.4)). The firing rate $m(t)$, in turn, depends on the boundary value of $\rho\left(V_{T}, g, t\right)$ through Eq. (4.8). In this sense, therefore, Eq. (4.3) is a nonlinear equation.

Since Eq. (4.3) is a nonlinear $(2+1)$-dimensional partial differential equation, clearly, we can achieve computational advantage by reducing the dynamics described by Eq. (4.3) to a $(1+1)$-dimensional effective dynamics. Through this reduction, we can also gain analytical insight into the neuronal network dynamics. In what follows, we discuss, in turn, two possible reductions: (i) in the limit of mean-driven dynamics and (ii) in the limit of fluctuation-driven dynamics.

4.2. Closure in the mean-driven limit. We define the mean-driven limit as the limit where $N \rightarrow \infty$, and $f \rightarrow 0, \nu_{0}(t) \rightarrow \infty$, but $f \nu_{0}(t)=$ finite. Obviously, in this limit, $\sigma_{g}^{2}(t)=0$ from Eq. (4.4b) and there will be no fluctuations in the input conductance for a neuron from either external input or the input from other neurons in the network. In this all-to-all network, the input conductance from the external input without fluctuations will be the same to all neurons, i.e., the effect of the second term $f \sum_{\mu} \delta\left(t-t_{\mu}^{i}\right)$ in Eq. (4.1b) is equivalent to a smooth input $f \nu_{0}(t)$, whereas the third term, which signifies the network interaction, in Eq. (4.1b), is equivalent to a smooth input $p S m(t)$ to a neuron from all other neurons in the network - the fluctuations have been scaled away by the factor $1 / \sqrt{N}$. Notice that, under these smooth conductance inputs without fluctuations, Eqs. (4.1) show that the voltages of neurons can move rapidly from the reset voltage $\varepsilon_{r}$ to the threshold $V_{T}$ and fire, which leads to the observation that the knowledge about the value of conductance of a neuron does not provide much information about the value of the voltage of the neuron statistically. Therefore, intuitively, the dynamics of conductance and voltage are uncorrelated in the mean-driven limit. If the dynamics of conductance and voltage are assumed to be statistically independent, i.e.,

$$
\rho(v, g, t)=\rho^{(v)}(v, t) \rho^{(g)}(g, t),
$$

then, the marginalization of Eq. (4.3) to $v$ and $g$ yields

$$
\begin{aligned}
\partial_{t} \rho^{(v)}(v) & =-\partial_{v} j_{V}(v) \\
\frac{d}{d t}\langle g\rangle & =-\frac{1}{\sigma}[\langle g\rangle-\bar{g}(t)]
\end{aligned}
$$

where

$$
j_{V}=-\left[\left(\frac{v-\varepsilon_{r}}{\tau}\right)+\langle g\rangle\left(\frac{v-\varepsilon_{E}}{\tau}\right)\right] \rho^{(v)}(v), \quad \text { and } \quad\langle g\rangle \equiv \int g \rho^{(g)}(g, t) d g .
$$






FIG. 4.1. Mean-driven Limit: Plotted here is the probability density function of a neuron's voltage. Solid line: Theoretic Prediction (4.10); Circles: Simulation of the original IESF neuronal network dynamics (4.1) in a steady state. Parameters are $N=1600, f=0.02 m s, \nu_{0}=20000 s^{-1}$, $S=0.05, \sigma=3 \mathrm{~ms}, \tau=20 \mathrm{~ms}$ and $p=1$. Inset: plotted is $1 / \rho(v)$, which is a linear function of $v$ from Eq. (4.10). We have scaled voltage units such that $\varepsilon_{r}=0, V_{T}=1$, and $\varepsilon_{E}=14 / 3$ [3]. This convention of units will be used throughout all the figures.

In the derivation, we have used the fact that the flux across the threshold $V_{T}$ is equal to the flux entering through the reset voltage $\varepsilon_{r}$, i.e.,

$$
\int_{\varepsilon_{r}}^{V_{T}} \partial_{v}\left\{\left[\left(\frac{v-\varepsilon_{r}}{\tau}\right)+g\left(\frac{v-\varepsilon_{E}}{\tau}\right)\right] \rho(v, g)\right\} d v=-J_{V}\left(V_{T}, g\right)+J_{V}\left(\varepsilon_{r}, g\right)=0
$$

by the boundary condition (4.6). Eqs. (4.9) are closed with respect to $\rho^{(v)}(v, t)$ and $\langle g\rangle(t)$ : Eq. $(4.9 \mathrm{a})$ is a $(1+1)$-dimensional PDE that describes the evolution of $\rho^{(v)}(v, t)$, whereas Eq. (4.9b) describes the evolution of the average conductance $\langle g\rangle(t)$.

For the case of time-homogeneous external input, i.e., $\nu_{0}(t)=\nu_{0}$, a constant, we can determine the steady-state firing rate as follows. First, note that the flux $j_{V}$ in Eq. (4.9a) is constant over $\left(\varepsilon_{r}, V_{T}\right)$ for a steady state. This constant can be determined by the flux at the boundary $V_{T}$, at which, the flux $j_{V}$ is equal to the firing rate. Therefore,

$$
m=j_{V}(v)=-\left[\left(\frac{v-\varepsilon_{r}}{\tau}\right)+\langle g\rangle\left(\frac{v-\varepsilon_{E}}{\tau}\right)\right] \rho^{(v)}(v) .
$$

For a steady state in a mean-driven regime, the probability density thus has the following form: 


$$
\rho^{(v)}(v)=-\frac{m \tau}{\left(v-\varepsilon_{r}\right)+\langle g\rangle\left(v-\varepsilon_{E}\right)} \quad \text { for } v \in\left[\varepsilon_{r}, V_{T}\right),
$$

and $\rho^{(v)}(v)=0$ for $v \notin\left[\varepsilon_{r}, V_{T}\right)$, where $\langle g\rangle=\bar{g}=f \nu_{0}+\bar{S} m$ by Eq. (4.9b). Using the normalization condition $\int_{\varepsilon_{r}}^{V_{T}} d v \rho^{(v)}(v)=1$ and noticing that the denominator in Eq. (4.10) must be negative to ensure $\rho^{(v)}(v) \geq 0$, we arrive at

$$
m=\left\{\begin{array}{cl}
\frac{1+\bar{g}}{\tau \log \left|\frac{\bar{g}\left(\varepsilon_{r}-\varepsilon_{E}\right)}{\left(V_{T}-\varepsilon_{r}\right)+\bar{g}\left(V_{T}-\varepsilon_{E}\right)}\right|}, & \text { if } \bar{g}>\frac{V_{T}-\varepsilon_{r}}{\varepsilon_{E}-V_{T}} \\
0, & \text { otherwise }
\end{array}\right.
$$

with $\bar{g}=f \nu_{0}+\bar{S} m$. By solving Eq. (4.11) for $m$, we can determine the firing rate. Note that Eq. (4.11) is not a new result and is commonly quoted in literature (see, for example, $[23,13])$. Here, we merely illustrate how to use the probability density description to reproduce this result.

Finally, we point out that, for an integrate-and-fire neuronal network operating in a mean-driven regime, the probability distribution for voltage is indeed well captured by Eq. (4.10), as is illustrated in Fig. (4.1).

4.3. Closure in fluctuation-driven regimes. In general, in contrast to the mean-driven limit, $g$ and $v$ are correlated. Stronger fluctuations in $g$ are expected to correlate with larger values of $v$. To describe this general dynamics, here, for the network described by Eq. (4.1), we derive a system of $(1+1)$-dimensional kinetic equations, which is one of our central results.

4.3.1. Dynamics of conductances. First, we discuss the dynamics of $\rho^{(g)}(g)=\int_{\varepsilon_{r}}^{V_{T}} \rho(v, g) d v$. Using the flux condition (4.6) at the boundary, we integrate Eq. (4.3) over $v$ to obtain

$$
\partial_{t} \rho^{(g)}(g)=\partial_{g}\left[\frac{1}{\sigma}(g-\bar{g}(t)) \rho^{(g)}(g)+\frac{\sigma_{g}^{2}(t)}{\sigma} \partial_{g} \rho^{(g)}(g)\right]
$$

for $g \in[0,+\infty)$. Clearly, in Eq. (4.12), the flux $J_{g}(g)=$ $-\left[\sigma^{-1}(g-\bar{g}(t)) \rho^{(g)}(g)+\sigma^{-1} \sigma_{g}^{2}(t) \partial_{g} \rho^{(g)}(g)\right]$ vanishes at $g=0$ and $g=\infty$. We note that, for time-homogeneous input, $\nu_{0}$ and $\sigma_{g}^{2}$ are constant, and the time-invariant solution can be approximated by the following Gaussian solution

$$
\rho^{(g)}(g)=\frac{1}{\sqrt{2 \pi} \sigma_{g}} \exp \left[-\frac{1}{2 \sigma_{g}^{2}}(g-\bar{g})^{2}\right]
$$

where $\bar{g}=\nu_{0} f+\bar{S} m$, under the condition $f \nu_{0}+\bar{S} m \gg \sigma_{g}$. Hence, the mean and variance of the conductance are

$$
\operatorname{mean}(g)=\bar{g}, \quad \operatorname{var}(g)=\sigma_{g}^{2},
$$

respectively. If the domain of $g$ were $(-\infty, \infty)$, then Eq. (4.13) would be exact. The numerical simulation of the full original I\&F dynamics (4.1) demonstrates that in certain regimes this approximate solution well captures the distribution of conductance as shown in Fig. (4.2). Note that the time-scale for the evolution of $\rho^{(g)}(g)$ 


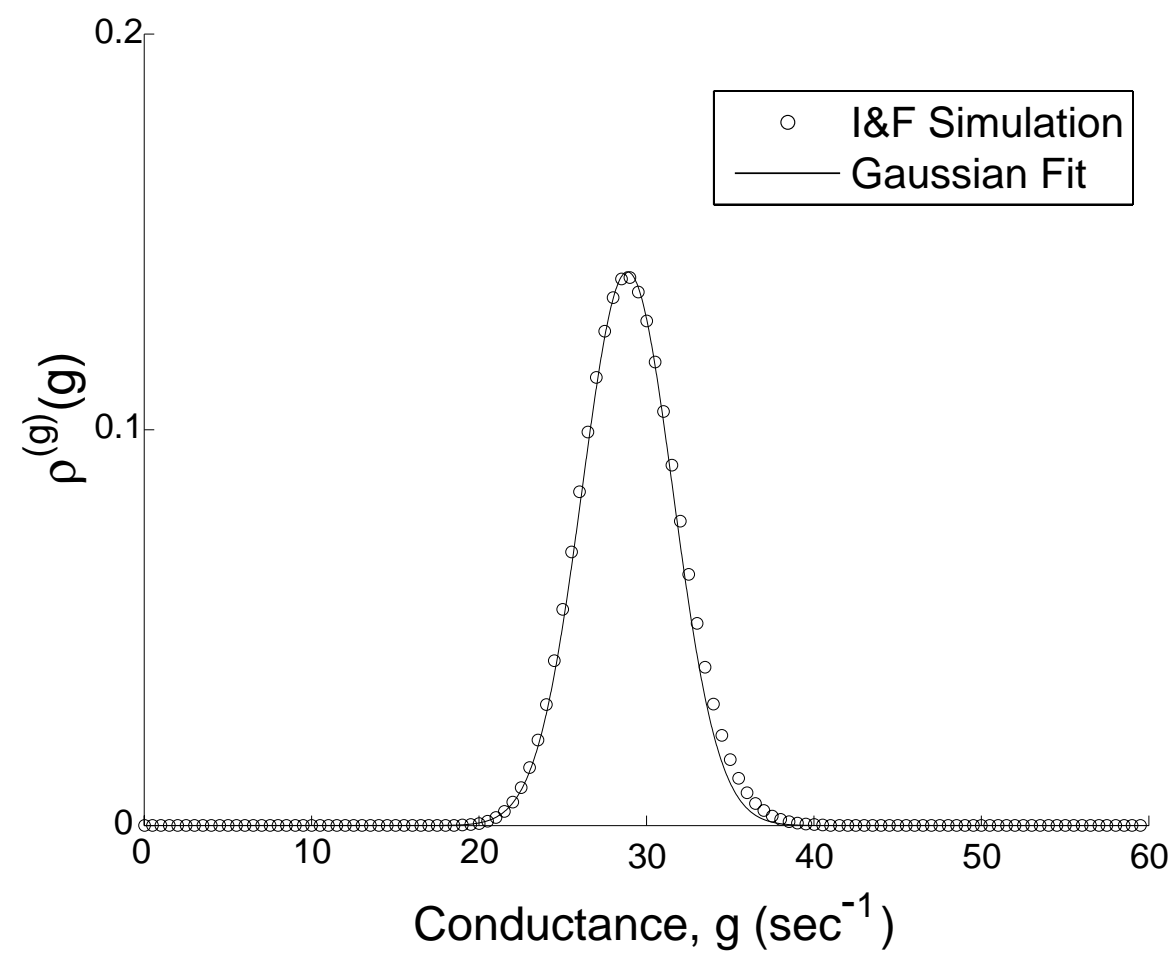

FIG. 4.2. Conductance Dynamics: Probability density function of $g$. Circles: Simulation of the original IEFF neuronal network dynamics (4.1); Solid line: Gaussian fit (4.13).

is $\sigma$. For sufficiently small $\sigma$, starting with any initial condition of $\rho^{(g)}(g)$, the solution rapidly converges to the approximate form in Eq. (4.13). If the time-scale of a time-dependent $\sigma_{g}^{2}(t)$ is much slower than $\sigma$ (which generally is the case for AMPA conductances, which are much faster than typical time-scales of stimulus input [38]), then, in the limit of $\sigma \rightarrow 0, \rho^{(g)}(g, t)$ is essentially slaved to the time-invariant solution that has the approximate form in Eq. (4.13). We note in passing that in the mean-driven limit, i.e., $\sigma_{g}^{2} \rightarrow 0$,

$$
\rho(g)=\delta\left(g-\nu_{0} f-\bar{S} m\right) .
$$

That is, the conductance does not fluctuate around the mean $\bar{g}=\nu_{0} f+\bar{S} m$, as is expected.

4.3.2. Dynamics of membrane potentials. Next, we project out the variable $g$ from Eq. (4.3). Define the conditional moments

$$
\mu_{1}(v)=\int_{0}^{\infty} g \rho(g \mid v) d g, \quad \mu_{2}(v)=\int_{0}^{\infty} g^{2} \rho(g \mid v) d g,
$$

where

$$
\rho(v, g)=\rho(g \mid v) \rho^{(v)}(v), \quad \text { and } \quad \rho^{(v)}(v)=\int_{0}^{\infty} \rho(v, g) d g .
$$


Integrating Eq. (4.3) over $g$ yields

$$
\partial_{t} \rho^{(v)}(v)=\partial_{v}\left\{\left[\left(\frac{v-\varepsilon_{r}}{\tau}\right)+\mu_{1}(v)\left(\frac{v-\varepsilon_{E}}{\tau}\right)\right] \rho^{(v)}(v)\right\},
$$

and multiplying Eq. (4.3) by $g$, then integrating over $g$ yields

$$
\begin{aligned}
\partial_{t}\left[\mu_{1}(v) \rho^{(v)}(v)\right]= & -\frac{1}{\sigma}\left[\mu_{1}(v)-\bar{g}(t)\right] \rho^{(v)}(v) \\
& +\partial_{v}\left[\left(\frac{v-\varepsilon_{r}}{\tau}\right) \mu_{1}(v) \rho^{(v)}(v)\right]+\partial_{v}\left[\left(\frac{v-\varepsilon_{E}}{\tau}\right) \mu_{2}(v) \rho^{(v)}(v)\right],
\end{aligned}
$$

where we have used Eq. (4.5b) and the boundary conditions (4.7). Using Eq. (4.14), we can cast Eq. (4.15) in the following form

$$
\begin{aligned}
\partial_{t} \mu_{1}(v)= & -\frac{1}{\sigma}\left(\mu_{1}(v)-\bar{g}(t)\right)+\left[\left(\frac{v-\varepsilon_{r}}{\tau}\right)+\mu_{1}(v)\left(\frac{v-\varepsilon_{E}}{\tau}\right)\right]\left[\partial_{v} \mu_{1}(v)\right] \\
& +\frac{\Sigma^{2}(v)}{\rho^{(v)}(v)} \partial_{v}\left[\left(\frac{v-\varepsilon_{E}}{\tau}\right) \rho^{(v)}(v)\right]+\left[\partial_{v} \Sigma^{2}(v)\right]\left(\frac{v-\varepsilon_{E}}{\tau}\right),
\end{aligned}
$$

where

$$
\Sigma^{2}(v) \equiv \mu_{2}(v)-\mu_{1}^{2}(v)
$$

is the conditional variance.

4.3.3. Closure. Equations (4.14) and (4.16) show that the evolution of $\rho^{(v)}(v)$ depends on the first conditional moment $\mu_{1}(v)$ and that the evolution of $\mu_{1}(v)$ depends on the second moment $\mu_{2}(v)$ through the conditional variance $\Sigma^{2}(v)$, $\cdots$. Therefore, projecting the dynamics (4.3) to the variable $v$ generates an infinite hierarchy of equations governing the conditional moments. Thus, the closure issue naturally arises, i.e., how to truncate this hierarchy to close equations to a lower order such that the truncated dynamics can still capture the essential dynamics of the system. Mathematically, a closure issue is often a coarse-graining issue, namely, whether there exits a scale below which the dynamics either by itself is sufficiently smooth (without much structure) or can be averaged out ${ }^{1}$. In our setting with excitatory neuronal networks, if the conditional variance is a sufficiently slowly varying function of $v$, then, we can naturally postulate the closure:

$$
\Sigma^{2}(v, t)=\sigma_{g}^{2}(t) .
$$

Therefore, we have $\partial_{v} \Sigma^{2}(v)=0$ and Eq. (4.16) becomes

$$
\begin{aligned}
\partial_{t} \mu_{1}(v)= & -\frac{1}{\sigma}\left[\mu_{1}(v)-\bar{g}(t)\right]+\frac{\sigma_{g}^{2}(t)}{\rho^{(v)}(v)} \partial_{v}\left[\left(\frac{v-\varepsilon_{E}}{\tau}\right) \rho^{(v)}(v)\right] \\
& +\left[\left(\frac{v-\varepsilon_{r}}{\tau}\right)+\mu_{1}(v)\left(\frac{v-\varepsilon_{E}}{\tau}\right)\right] \partial_{v} \mu_{1}(v) .
\end{aligned}
$$

\footnotetext{
${ }^{1}$ Note that this coarse-graining in dynamics is different from the spatial coarse-graining procedure we described in Introduction about partitioning a region of cortex into coarse-grained patches.
} 
Now Eqs. (4.14) and (4.18) are closed with respect to $\rho^{(v)}(v)$ and $\mu_{1}(v)$. In summary,

$$
\begin{aligned}
\partial_{t} \rho^{(v)}(v)= & \partial_{v}\left\{\left[\left(\frac{v-\varepsilon_{r}}{\tau}\right)+\mu_{1}(v)\left(\frac{v-\varepsilon_{E}}{\tau}\right)\right] \rho^{(v)}(v)\right\}, \\
\partial_{t} \mu_{1}(v)= & -\frac{1}{\sigma}\left[\mu_{1}(v)-\bar{g}(t)\right]+\frac{\sigma_{g}^{2}(t)}{\rho^{(v)}(v)} \partial_{v}\left[\left(\frac{v-\varepsilon_{E}}{\tau}\right) \rho^{(v)}(v)\right] \\
& +\left[\left(\frac{v-\varepsilon_{r}}{\tau}\right)+\mu_{1}(v)\left(\frac{v-\varepsilon_{E}}{\tau}\right)\right] \partial_{v} \mu_{1}(v)
\end{aligned}
$$

with

$$
\begin{aligned}
\bar{g}(t) & \equiv f \nu_{0}(t)+\bar{S} m(t), \\
\sigma_{g}^{2}(t) & \equiv \frac{1}{2 \sigma}\left[f^{2} \nu_{0}(t)+\frac{\bar{S}^{2}}{p N} m(t)\right],
\end{aligned}
$$

constitute our key result: kinetic equations for the dynamics of coupled excitatory neuronal networks. From Eq. (4.19a), the corresponding probability flux clearly is

$$
\mathcal{J}_{V}(v, t)=-\left[\left(\frac{v-\varepsilon_{r}}{\tau}\right)+\mu_{1}(v)\left(\frac{v-\varepsilon_{E}}{\tau}\right)\right] \rho^{(v)}(v) .
$$

Therefore, the population-averaged firing rate per neuron is determined by the flux (4.20) at the threshold:

$$
m(t)=\mathcal{J}_{V}\left(V_{T}, t\right) .
$$

4.3.4. Boundary conditions. Now we discuss how to derive boundary conditions for kinetic equations (4.19). Note the probability flux (4.5a) along the $v$-direction satisfies the boundary condition (4.6). Therefore,

$$
\int_{0}^{\infty} J_{V}\left(V_{T}, g\right) d g=\int_{0}^{\infty} J_{V}\left(\varepsilon_{r}, g\right) d g
$$

which leads to

$$
\left[\left(V_{T}-\varepsilon_{r}\right)+\left(V_{T}-\varepsilon_{E}\right) \mu_{1}\left(V_{T}\right)\right] \rho^{(v)}\left(V_{T}\right)=\left(\varepsilon_{r}-\varepsilon_{E}\right) \mu_{1}\left(\varepsilon_{r}\right) \rho^{(v)}\left(\varepsilon_{r}\right) .
$$

Furthermore, for the quantity,

$$
\eta(v)=\int_{0}^{\infty} g J_{V}(v, g) d g
$$

using the closure (4.17), it is easy to show that

$$
\eta(v)=\mathcal{J}_{V}(v, t) \mu_{1}(v)-\sigma_{g}^{2}\left(\frac{v-\varepsilon_{E}}{\tau}\right) \rho^{(v)}(v)
$$

with $\mathcal{J}_{V}(v, t)$ as in Eq. (4.20). The boundary condition (Eq. (4.6)) entails

$$
\eta\left(V_{T}\right)=\eta\left(\varepsilon_{r}\right),
$$




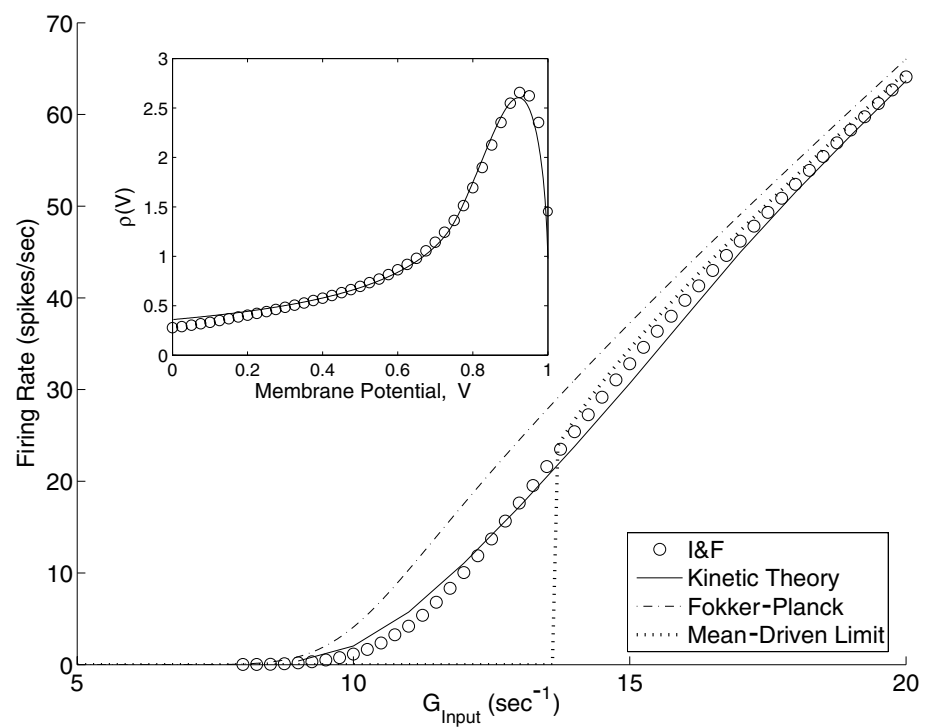

FIG. 4.3. Steady State Accuracy of Kinetic Theory for Fluctuation-Dominated Dynamics: The population-averaged firing rate per neuron $m$ as a function of mean input conductance $G_{\text {input }}=$ $f \nu_{0}$. Inset: probability density function of the membrane potential. Circles: Simulation of the original purely excitatory IEFF neuronal dynamics (4.1) in a statistical steady state; Solid line: Kinetic Theory (4.19)with BCs derived from Eqs. (4.21) and (4.22); Dot-dashed line: FokkerPlanck Equation (4.24); Dotted line: Mean-Driven Limit (4.11). Parameters: $f=0.2 \mathrm{~ms}, S=0.05$, $\sigma=3 \mathrm{~ms}, \tau=20 \mathrm{~ms}, N=300$, and $p=0.25 . \quad \varepsilon_{r}=0, V_{T}=1$, and $\varepsilon_{E}=14 / 3 . \quad\left(\nu_{0}=1200 \mathrm{~s}^{-1}\right.$ for the inset.)

i.e.,

$$
\begin{aligned}
& \mathcal{J}_{V}\left(V_{T}, t\right) \mu_{1}\left(V_{T}\right)-\sigma_{g}^{2}\left(\frac{V_{T}-\varepsilon_{E}}{\tau}\right) \rho^{(v)}\left(V_{T}\right) \\
= & \mathcal{J}_{V}\left(\varepsilon_{r}, t\right) \mu_{1}\left(\varepsilon_{r}\right)-\sigma_{g}^{2}\left(\frac{\varepsilon_{r}-\varepsilon_{E}}{\tau}\right) \rho^{(v)}\left(\varepsilon_{r}\right)
\end{aligned}
$$

Note that $\mathcal{J}_{V}\left(V_{T}, t\right)=\mathcal{J}_{V}\left(\varepsilon_{r}, t\right)=m(t)$. Hence,

$$
\tau m(t)\left[\mu_{1}\left(V_{T}\right)-\mu_{1}\left(\varepsilon_{r}\right)\right]=\sigma_{g}^{2}\left[\left(V_{T}-\varepsilon_{E}\right) \rho^{(v)}\left(V_{T}\right)-\left(\varepsilon_{r}-\varepsilon_{E}\right) \rho^{(v)}\left(\varepsilon_{r}\right)\right] .
$$

Eqs. (4.21) and (4.22) constitute nonlinear boundary conditions for the kinetic equations (4.19).

4.4. Accuracy of kinetic theory. As pointed out in Ref. [17], our kinetic theory can reproduce the voltage distribution and firing rate very well. Figure (4.3) illustrates a comparison between the predictions of our kinetic theory and the full numerical simulation of the original I\&F purely excitatory neuronal networks (4.1) in a steady state. Note that even when the conductance mean input $G_{\text {input }}=f \nu_{0}$ is not sufficiently strong to force a neuron to fire in the mean-driven limit (for example, for the values of $G_{\text {input }}<13.6$ in Eq. (4.11)), our kinetic theory captures the fluctuation-induced firing very well, as shown in Fig. (4.3). Further, here we show that the time-dependent solutions of our kinetic theory can also capture very well the 

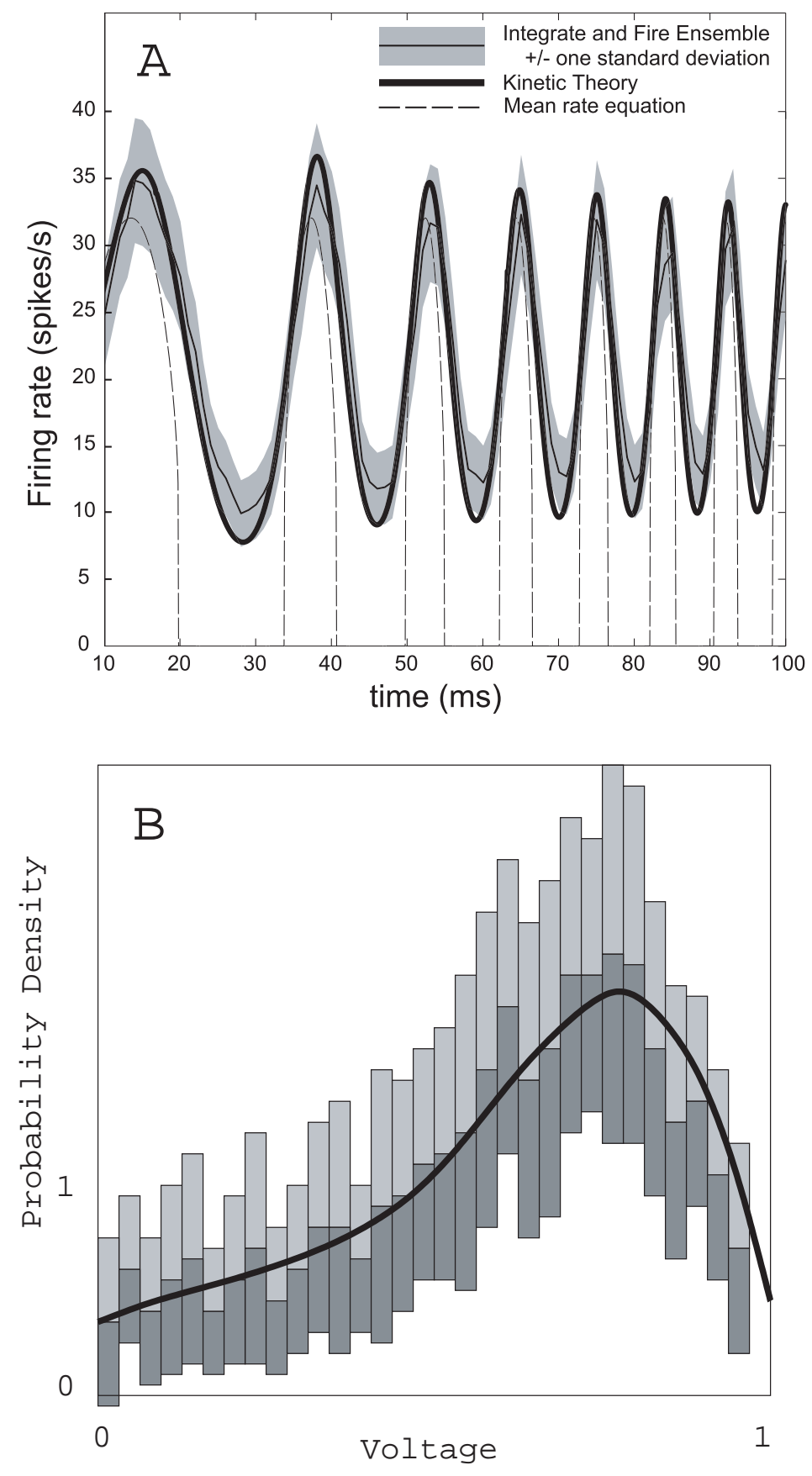

Fig. 4.4. Dynamical Accuracy of Kinetic Theory in a Fluctuation-Dominated Dynamics: Plotted for a network (4.1) of $N=100$ purely excitatory IEF neurons driven by a time-varying input rate $\nu_{0}(t)=\nu_{a} \exp \left[0.25 \sin \left(2 \pi t / s+(2 \pi t / s)^{2}\right)\right]$, where $\nu_{a}=0.5$ spikes $/ \mathrm{ms}, s=100 \mathrm{~ms}$, is (A) The firing rate $m(t)$ (Thin solid line: simulation result averaged over an ensemble of $10^{4}$ identically structured networks. The firing rate is measured using a bin size of 1 msec. The upper and lower boundaries of the gray area mark the values one-standard deviation away from the mean, measured from the ensemble of the $10^{4}$ networks. Thick solid line: Kinetic theory (4.19). Dashed line: Meandriven limit (4.11)); (B) Instantaneous probability density function of the membrane potential at time $t=79.625 \mathrm{~ms}$ (The upper and lower boundaries of the gray area mark the values one-standard deviation away from the mean, measured from the ensemble of the $10^{4}$ networks. Thick solid line: Kinetic theory (4.19)). Parameters: $f=0.5 \mathrm{~ms}, \tau=20 \mathrm{~ms}$ and $\sigma=0.1 \mathrm{~ms}, S=0.125$ and $p=0$. 
original dynamics of the full I\&F neuronal network (4.1). Figure (4.4) clearly demonstrates this dynamic accuracy of our kinetic theory. It is important to emphasize that our kinetic theory can capture fluctuation-dominated dynamics accurately, including fluctuations induced by finite sizes of networks.

We note that under the mean-driven limit, i.e., $N \rightarrow \infty$, and $f \rightarrow 0, \nu_{0} \rightarrow \infty$, with $f \nu_{0}=$ finite, for which $\sigma_{g}^{2}=0$, the kinetic equations (4.19) recover the mean-driven equations (4.9) with $\langle g\rangle$ being slaved to $\bar{g}$. Therefore, our kinetic theory has a wide range of validity - from the mean-driven regimes to the fluctuation-dominate regime. We will further discuss the issue of $v-g$ correlation and fluctuations below.

4.5. Fokker-Planck Equation. The kinetic equations (Eqs (4.19)) can be viewed as asymptotic equations for describing the neuronal network dynamics for a small but finite $\sigma$. Now we discuss the $\sigma \rightarrow 0$ limit of kinetic equations (Eqs (4.19)). Note that

$$
\sigma \sigma_{g}^{2}(t)=\frac{1}{2}\left[f^{2} \nu_{0}(t)+\frac{\bar{S}^{2}}{N p} m(t)\right] \sim \mathcal{O}(1) .
$$

As $\sigma \rightarrow 0$, Eq. (4.19b) reduces to

$$
\begin{aligned}
\mu_{1}(v) & =\bar{g}(t)+\frac{\sigma \sigma_{g}^{2}}{\rho^{(v)}(v)} \partial_{v}\left[\left(\frac{v-\varepsilon_{E}}{\tau}\right) \rho^{(v)}(v)\right] \\
& =\bar{g}(t)+\frac{\sigma \sigma_{g}^{2}}{\tau}+\frac{\sigma \sigma_{g}^{2}}{\rho^{(v)}(v)}\left(\frac{v-\varepsilon_{E}}{\tau}\right) \partial_{v} \rho^{(v)}(v) .
\end{aligned}
$$

This expression of $\mu_{1}(v)$ makes transparent the meaning of corrections to the first term in Eq. (4.23), i.e., $\bar{g}(t)$, which describes the mean-driven, uncorrelated limit.

1. Due to the spread with size $\sigma_{g}$ of $g$, there is a fluctuation correction $\frac{\sigma \sigma_{g}^{2}}{\tau}$ to $\bar{g}(t)$. This fluctuation correction will effectively lower the threshold.

2. Fluctuations induce a correlation correction between $g$ and $v$ as signified by the last term in Eq. (4.23). As will be seen below (Eq. (4.27)), $\partial_{v} \rho^{(v)}(v)<$ 0 at the threshold $V_{T}$, the correlation correction is thus positive near the threshold. Therefore, the net effect of this last term also lowers the effective threshold for firing.

The combined effects of fluctuations is to effectively lower the firing threshold. Therefore, even if $\bar{g}$ is subthreshold, neurons in the network can still fire.

Finally, substituting Eq. (4.23) into Eq. (4.19a) yields the following FokkerPlanck equation,

$$
\partial_{t} \rho^{(v)}(v)=\partial_{v}\left\{\left[\left(\frac{v-\varepsilon_{r}}{\tau}\right)+\gamma(t)\left(\frac{v-\varepsilon_{E}}{\tau}\right)\right] \rho^{(v)}(v)+\sigma \sigma_{g}^{2}(t)\left(\frac{v-\varepsilon_{E}}{\tau}\right)^{2} \partial_{v} \rho^{(v)}(v)\right\}
$$

where $\gamma(t) \equiv \bar{g}(t)+\sigma \sigma_{g}^{2}(t) / \tau$. Clearly, we have condition that $\int_{\varepsilon_{r}}^{\varepsilon_{E}} \rho^{(v)}(v) d v=1$. The probability flux in Eq. (4.24) is

$$
J_{\mathrm{FP}}(v, t)=-\left[\left(\frac{v-\varepsilon_{r}}{\tau}\right)+\gamma(t)\left(\frac{v-\varepsilon_{E}}{\tau}\right)\right] \rho^{(v)}(v)-\sigma \sigma_{g}^{2}(t)\left(\frac{v-\varepsilon_{E}}{\tau}\right)^{2} \partial_{v} \rho^{(v)}(v) .
$$

It is easy to verify that, in the absence of network couplings, i.e., $S=0$, Eq. (4.24) reduces to Eq. (3.12) (Note that $\varepsilon_{E}$ is set to 0 in Eq. (3.12)). 
We observe that Eq. (4.24) is derived by taking the $\sigma \rightarrow 0$ limit of the kinetic equations (4.19), which is derived with a finite $\sigma$ under the diffusion approximation (4.3), whereas Eq. (3.12) is derived under the diffusion approximation of Eq. (3.7), which is an evolution equation for $\rho(v)$ with an infinitely fast (i.e., $\sigma=0)$ conductance. There is a certain internal consistency reflected in the fact that these two limiting procedures yield the same Fokker-Planck equation. This consistency further stresses the significance of the Stratonovich interpretation mentioned above as a correct physical interpretation of stochastic dynamics (3.13).

Finally, we discuss the boundary conditions for solving Eq. (4.24). There is a subtlety in choosing boundary conditions: One way is to use the $\sigma \rightarrow 0$ limit of boundary conditions derived for the kinetic equation. In this limit, Eqs. (4.21) and (4.22) become

$$
\begin{gathered}
J_{\mathrm{FP}}\left(\varepsilon_{r}, t\right)=J_{\mathrm{FP}}\left(V_{T}, t\right)=m(t), \\
\left(V_{T}-\varepsilon_{E}\right) \rho^{(v)}\left(V_{T}\right)=\left(\varepsilon_{r}-\varepsilon_{E}\right) \rho^{(v)}\left(\varepsilon_{r}\right),
\end{gathered}
$$

respectively. However, there is another way of prescribing boundary conditions if we interpret the $\sigma \rightarrow 0$ limit as the following physical process: the conductance has a vanishing decay time and upon receiving an input spike, a neuron still receives a finite but very small jump (diffusion approximation) in $v$. When near the threshold, it jumps across the threshold, and immediately resets, i.e., no longer dwelling near the threshold as with the case of finite $\sigma$ (With a finite $\sigma$, this dwelling is possible due to a slow upward streaming of $v$ under the network dynamics (4.1) ). Therefore, there is no accumulation of neuron population at the threshold. Hence, we can postulate the boundary conditions

$$
\begin{aligned}
\rho^{(v)}\left(V_{T}, t\right) & =0, \\
J_{\mathrm{FP}}\left(\varepsilon_{r}, t\right) & =J_{\mathrm{FP}}\left(V_{T}, t\right),
\end{aligned}
$$

the second of which simply reflects the fact that the neurons that just fired all enter through the reset potential. The firing rate now is expressed as

$$
\begin{aligned}
m(t) & =J_{\mathrm{FP}}\left(V_{T}, t\right) \\
& =-\left.\sigma \sigma_{g}^{2}\left(\frac{V_{T}-\varepsilon_{E}}{\tau}\right)^{2} \partial_{v} \rho^{(v)}(v)\right|_{v=V_{T}} .
\end{aligned}
$$

In general, $m(t)>0$, therefore,

$$
\left.\partial_{v} \rho^{(v)}(v)\right|_{v=V_{T}}<0
$$

We note that these two different boundary conditions reflect the difference in fundamental "microscopic" I\&F neurons. Boundary condition (Eq. (4.25)) corresponds to an I\&F neuron with a finite $\sigma$, as $\sigma \rightarrow 0$, whereas boundary condition (Eq. (4.26)) corresponds to an I\&F neuron with $\sigma=0$. In some parameter regimes, these two boundary conditions (i.e., Eq. (4.25) vs. Eq. (4.26)) produce very similar solutions of $\rho^{(v)}(v)$ as illustrated in Fig. (4.5). In terms of practical purposes of modeling qualitative statistical behavior, either of the boundary conditions can be used in the limit of $\sigma \rightarrow 0$. 


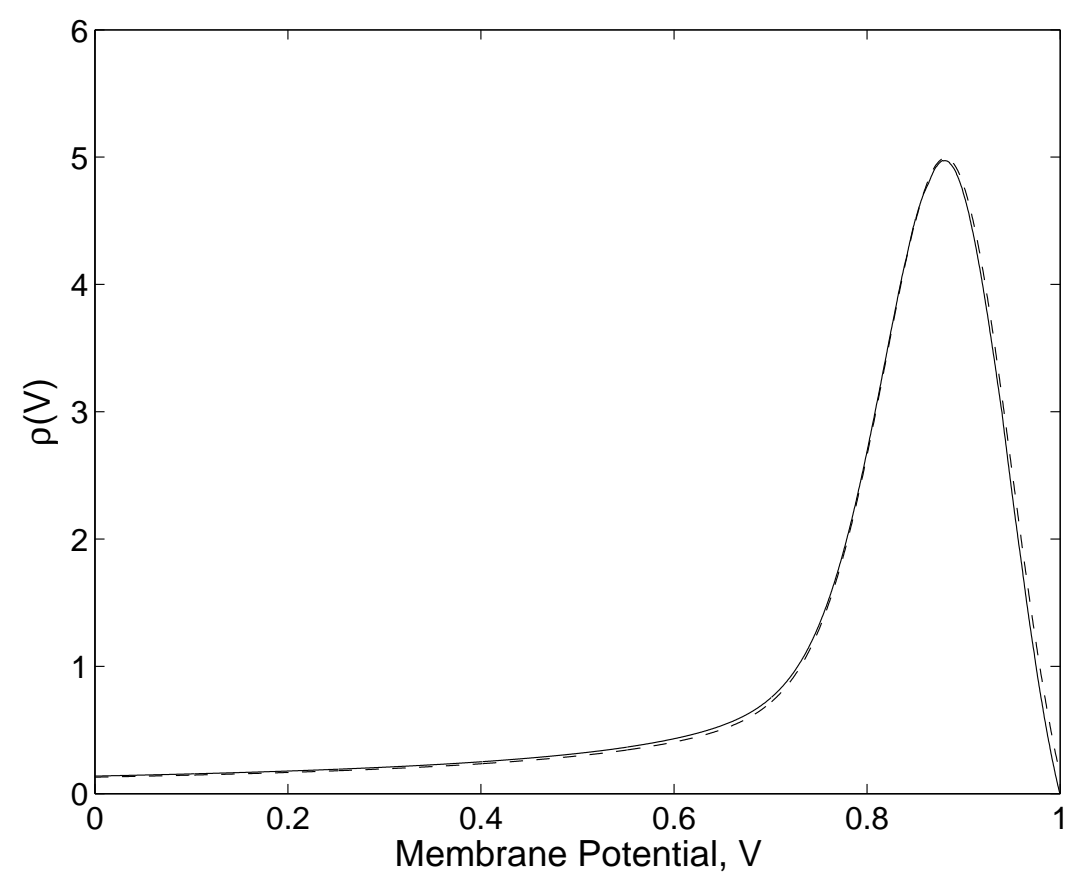

FIG. 4.5. Comparison of Boundary Conditions: Solutions of the Fokker-Planck equation (4.24) solved with boundary conditions (4.26) (solid), and boundary conditions (4.25) (dashed), with corresponding $m=7.05$ and $m=7.65$, respectively. $f=0.6 m s, \nu_{0}=4000 \mathrm{~s}^{-1}, N=75$ and $S=0.01$.

\section{Networks with excitatory and inhibitory neurons}

In this section, we extend our kinetic theory to the case of the coupled I\&F dynamics (2.1) with both excitatory and inhibitory neurons. The detailed description of the network is already given in Section 2. Following the similar arguments in previous sections, the probability density function we are interested in is now defined by

$$
\rho_{\lambda}\left(v, g_{E}, g_{I}, t\right)=\mathbb{E}\left[\frac{1}{N_{\lambda}} \sum_{i=1}^{N_{\lambda}} \delta\left(v-V_{i}^{\lambda}(t)\right) \delta\left(g_{E}-G_{i}^{\lambda E}(t)\right) \delta\left(g_{I}-G_{i}^{\lambda I}(t)\right)\right]
$$

for the $\lambda$-population, $\lambda=E, I$, labeling excitation and inhibition, where $\mathbb{E}(\cdot)$ is the expectation with respect to all possible sets of independent realizations of the input Poisson spike process in an ensemble of identically structured networks. Denote $\rho_{\lambda} \equiv$ $\rho_{\lambda}\left(v, g_{E}, g_{I}, t\right), \bar{S}_{\lambda \lambda^{\prime}}=p S_{\lambda \lambda^{\prime}}$. Assuming (i) $N_{E}, N_{I} \gg 1$, and (ii) the Poisson property for summed spike trains from all other neurons in the network, a similar derivation to what is described in Appendix B leads to

$$
\begin{aligned}
\partial_{t} \rho_{\lambda}= & \partial_{v}\left\{\left[\left(\frac{v-\varepsilon_{r}}{\tau}\right)+g_{E}\left(\frac{v-\varepsilon_{E}}{\tau}\right)+g_{I}\left(\frac{v-\varepsilon_{I}}{\tau}\right)\right] \rho_{\lambda}\right\}+\partial_{g_{E}}\left(\frac{g_{E}}{\sigma_{E}} \rho_{\lambda}\right)+\partial_{g_{I}}\left(\frac{g_{I}}{\sigma_{I}} \rho_{\lambda}\right) \\
& +\nu_{0 E}(t)\left[\rho_{\lambda}\left(v, g_{E}-\frac{f_{E}}{\sigma_{E}}, g_{I}\right)-\rho_{\lambda}\left(v, g_{E}, g_{I}\right)\right]
\end{aligned}
$$




$$
\begin{aligned}
& +\nu_{0 I}(t)\left[\rho_{\lambda}\left(v, g_{E}, g_{I}-\frac{f_{I}}{\sigma_{I}}\right)-\rho_{\lambda}\left(v, g_{E}, g_{I}\right)\right] \\
& +p m_{E}(t) N_{E}\left[\rho_{\lambda}\left(v, g_{E}-\frac{\bar{S}_{\lambda E}}{p N_{E} \sigma_{E}}, g_{I}\right)-\rho_{\lambda}\left(v, g_{E}, g_{I}\right)\right] \\
& +p m_{I}(t) N_{I}\left[\rho_{\lambda}\left(v, g_{E}, g_{I}-\frac{\bar{S}_{\lambda I}}{p N_{I} \sigma_{I}}\right)-\rho_{\lambda}\left(v, g_{E}, g_{I}\right)\right]
\end{aligned}
$$

for $v \in\left(\varepsilon_{I}, V_{T}\right), g_{E} \in(0, \infty)$, and $g_{I} \in(0, \infty)$. Note that the probability flux for Eq. (5.1) along the $v$-direction is

$$
J_{\lambda}\left(v, g_{E}, g_{I}, t\right)=-\left[\left(\frac{v-\varepsilon_{r}}{\tau}\right)+g_{E}\left(\frac{v-\varepsilon_{E}}{\tau}\right)+g_{I}\left(\frac{v-\varepsilon_{I}}{\tau}\right)\right] \rho_{\lambda}\left(v, g_{E}, g_{I}, t\right) .
$$

Therefore, the population-average firing rate per neuron for the $\lambda$-population is

$$
m_{\lambda}(t)=\int J_{\lambda}\left(V_{T}, g_{E}, g_{I}, t\right) d g_{E} d g_{I}
$$

At $v=\varepsilon_{r}$, we have the flux condition

$$
J_{\lambda}\left(\varepsilon_{r}^{+}, g_{E}, g_{I}, t\right)=J_{\lambda}\left(\varepsilon_{r}^{-}, g_{E}, g_{I}, t\right)+J_{\lambda}\left(V_{T}, g_{E}, g_{I}, t\right)
$$

where $J_{\lambda}\left(V_{T}, g_{E}, g_{I}, t\right)$ is the probability flux of neurons at $v=V_{T}$ for the population $\lambda$ - neurons that just fired come out from the reset voltage (Note that the reset dynamics we are considering is instantaneous, i.e., no refractory period). Clearly, we have the flux condition at the boundary $v=\varepsilon_{I}$,

$$
J_{\lambda}\left(\varepsilon_{I}, g_{E}, g_{I}, t\right)=0
$$

since no neurons have a voltage below the inhibitory reversal potential.

A diffusion approximation reduces Eq. (5.1) to

$$
\begin{aligned}
\partial_{t} \rho_{\lambda}= & \partial_{v}\left\{\left[\left(\frac{v-\varepsilon_{r}}{\tau}\right)+g_{E}\left(\frac{v-\varepsilon_{E}}{\tau}\right)+g_{I}\left(\frac{v-\varepsilon_{I}}{\tau}\right)\right] \rho_{\lambda}\right\} \\
& +\partial_{g_{E}}\left\{\frac{1}{\sigma_{E}}\left[\left(g_{E}-\bar{g}_{\lambda E}(t)\right) \rho_{\lambda}+\sigma_{\lambda E}^{2} \partial_{g_{E}} \rho_{\lambda}\right]\right\} \\
& +\partial_{g_{I}}\left\{\frac{1}{\sigma_{I}}\left[\left(g_{I}-\bar{g}_{\lambda I}(t)\right) \rho_{\lambda}+\sigma_{\lambda I}^{2} \partial_{g_{I}} \rho_{\lambda}\right]\right\}
\end{aligned}
$$

with

$$
\begin{array}{cc}
\bar{g}_{\lambda E}(t)=f_{E} \nu_{0 E}(t)+\bar{S}_{\lambda E} m_{E}(t), & \bar{g}_{\lambda I}(t)=f_{I} \nu_{0 I}(t)+\bar{S}_{\lambda I} m_{I}(t) \\
\sigma_{\lambda E}^{2}=\frac{1}{2 \sigma_{E}}\left[f_{E}^{2} \nu_{0 E}(t)+\frac{\bar{S}_{\lambda E}^{2}}{p N_{E}} m_{E}(t)\right], & \sigma_{\lambda I}^{2}=\frac{1}{2 \sigma_{I}}\left[f_{I}^{2} \nu_{0 I}(t)+\frac{\bar{S}_{\lambda I}^{2}}{p N_{I}} m_{I}(t)\right] .
\end{array}
$$

We note that, again, through Eqs. (5.2) and (5.6), Eq. (5.5) is a nonlinear partial differential equation over space $\left(v, g_{E}, g_{I}\right)$. Our aim is to extend our closure to derive a system of $(1+1)$-dimensional kinetic equations to capture the essential coarse-grained dynamics of Eq. (5.1). 
5.1. Dynamics of conductances. First, we discuss the dynamics of conductances. Using flux boundary conditions (5.3) and (5.4), we have

$$
\begin{aligned}
& -\int_{\varepsilon_{I}}^{V_{T}} d v \partial_{v}\left\{\left[\left(\frac{v-\varepsilon_{r}}{\tau}\right)+g_{E}\left(\frac{v-\varepsilon_{E}}{\tau}\right)+g_{I}\left(\frac{v-\varepsilon_{I}}{\tau}\right)\right] \rho_{\lambda}\right\} \\
= & J_{\lambda}\left(V_{T}, g_{E}, g_{I}, t\right)-J_{\lambda}\left(\varepsilon_{r}^{+}, g_{E}, g_{I}, t\right)+J_{\lambda}\left(\varepsilon_{r}^{-}, g_{E}, g_{I}, t\right)-J_{\lambda}\left(\varepsilon_{I}, g_{E}, g_{I}, t\right) \\
= & 0 .
\end{aligned}
$$

Therefore, integration of Eq. (5.5) over $v$ from $\varepsilon_{I}$ to $V_{T}$ leads to

$$
\begin{aligned}
\partial_{t} \tilde{\rho}_{\lambda}\left(g_{E}, g_{I}\right)= & \partial_{g_{E}}\left\{\frac{1}{\sigma_{E}}\left[\left(g_{E}-\bar{g}_{\lambda E}(t)\right)+\sigma_{\lambda E}^{2} \partial_{g_{E}}\right] \tilde{\rho}_{\lambda}\left(g_{E}, g_{I}\right)\right\} \\
& +\partial_{g_{I}}\left\{\frac{1}{\sigma_{I}}\left[\left(g_{I}-\bar{g}_{\lambda I}(t)\right)+\sigma_{\lambda I}^{2} \partial_{g_{I}}\right] \tilde{\rho}_{\lambda}\left(g_{E}, g_{I}\right)\right\}
\end{aligned}
$$

where $\tilde{\rho}_{\lambda}\left(g_{E}, g_{I}\right)=\int_{\varepsilon_{I}}^{V_{T}} d v \rho_{\lambda}\left(v, g_{E}, g_{I}, t\right)$. Using the fact that the flux along $g_{I}$ vanishes at $g_{I}=0$ and $g_{I}=\infty$, and further integrating Eq. (5.7) over $g_{I}$ yields

$$
\partial_{t} \rho_{\lambda}^{(\mathrm{E})}\left(g_{E}\right)=\partial_{g_{E}}\left\{\frac{1}{\sigma_{E}}\left[\left(g_{E}-\bar{g}_{\lambda E}(t)\right)+\sigma_{\lambda E}^{2} \partial_{g_{E}}\right] \rho_{\lambda}^{(\mathrm{E})}\left(g_{E}\right)\right\},
$$

where $\rho_{\lambda}^{(\mathrm{E})}\left(g_{E}\right)=\int_{0}^{\infty} d g_{I} \tilde{\rho}_{\lambda}\left(g_{E}, g_{I}\right)$. Similarly, we obtain

$$
\partial_{t} \rho_{\lambda}^{(\mathrm{I})}\left(g_{I}\right)=\partial_{g_{I}}\left\{\frac{1}{\sigma_{I}}\left[\left(g_{I}-\bar{g}_{\lambda I}(t)\right)+\sigma_{\lambda I}^{2} \partial_{g_{I}}\right] \rho_{\lambda}^{(\mathrm{I})}\left(g_{I}\right)\right\} .
$$

Next, we discuss the correlation between the inhibitory conductance and the excitatory conductance. For the moments defined by

$$
\begin{aligned}
\left\langle g_{E} g_{I}\right\rangle & =\int_{0}^{\infty} d g_{E} \int_{0}^{\infty} d g_{I} g_{E} g_{I} \tilde{\rho}_{\lambda}\left(g_{E}, g_{I}\right), \\
\left\langle g_{E}\right\rangle & =\int_{0}^{\infty} d g_{E} \int_{0}^{\infty} d g_{I} g_{E} \tilde{\rho}_{\lambda}\left(g_{E}, g_{I}\right),\left\langle g_{I}\right\rangle=\int_{0}^{\infty} d g_{E} \int_{0}^{\infty} d g_{I} g_{I} \tilde{\rho}_{\lambda}\left(g_{E}, g_{I}\right) .
\end{aligned}
$$

Eqs. (5.7), (5.8), and (5.9) lead to

$$
\begin{gathered}
\frac{d}{d t}\left\langle g_{E} g_{I}\right\rangle=-\left(\frac{1}{\sigma_{E}}+\frac{1}{\sigma_{I}}\right)\left\langle g_{E} g_{I}\right\rangle+\frac{1}{\sigma_{E}}\left\langle g_{I}\right\rangle \bar{g}_{\lambda E}(t)+\frac{1}{\sigma_{I}}\left\langle g_{E}\right\rangle \bar{g}_{\lambda I}(t), \\
\frac{d}{d t}\left\langle g_{E}\right\rangle=-\frac{1}{\sigma_{E}}\left[\left\langle g_{E}\right\rangle-\bar{g}_{\lambda E}(t)\right] \\
\frac{d}{d t}\left\langle g_{I}\right\rangle=-\frac{1}{\sigma_{I}}\left[\left\langle g_{I}\right\rangle-\bar{g}_{\lambda I}(t)\right]
\end{gathered}
$$

respectively. Then, the correlation,

$$
C\left(g_{E}, g_{I}\right) \equiv\left\langle g_{E} g_{I}\right\rangle-\left\langle g_{E}\right\rangle\left\langle g_{I}\right\rangle,
$$

can be shown to follow the dynamics

$$
\frac{d}{d t} C\left(g_{E}, g_{I}\right)=-\left(\frac{1}{\sigma_{E}}+\frac{1}{\sigma_{I}}\right) C\left(g_{E}, g_{I}\right),
$$


using Eqs. (5.10), (5.11), and (5.12). Even if there is an initial correlation in the initial population ensemble, according to Eq. (5.13), the correlation will quickly decay over the time-scale $\sigma_{E} \sigma_{I} /\left(\sigma_{E}+\sigma_{I}\right)$. Therefore, the inhibitory conductance can be regarded as uncorrelated with the excitatory conductance.

Similar to the case of a purely excitatory neuronal network, if both $\sigma_{E}$ and $\sigma_{I}$ are considered to be small, then the time-invariant solutions of Eqs. (5.8) and (5.9) are nearly Gaussian with means and variances given by Eqs. (5.6).

5.2. Closure in fluctuation-driven dynamic regimes. To project the dynamics (5.5) to $v$-space, we consider the evolution equations governing the conditional moments defined by

$$
\mu_{\lambda E}(v)=\int_{0}^{\infty} d g_{E} \int_{0}^{\infty} d g_{I} g_{E} \rho_{\lambda}\left(g_{E}, g_{I} \mid v\right), \quad \mu_{\lambda I}(v)=\int_{0}^{\infty} d g_{E} \int_{0}^{\infty} d g_{I} g_{I} \rho_{\lambda}\left(g_{E}, g_{I} \mid v\right)
$$

$\mu_{\lambda E}^{(2)}(v)=\int_{0}^{\infty} d g_{E} \int_{0}^{\infty} d g_{I} g_{E}^{2} \rho_{\lambda}\left(g_{E}, g_{I} \mid v\right), \quad \mu_{\lambda I}^{(2)}(v)=\int_{0}^{\infty} d g_{E} \int_{0}^{\infty} d g_{I} g_{I}^{2} \rho_{\lambda}\left(g_{E}, g_{I} \mid v\right)$ $\mu_{\lambda E I}^{(2)}(v)=\int_{0}^{\infty} d g_{E} \int_{0}^{\infty} d g_{I} g_{E} g_{I} \rho_{\lambda}\left(g_{E}, g_{I} \mid v\right)$

where $\rho_{\lambda}\left(v, g_{E}, g_{I}, t\right)=\rho_{\lambda}\left(g_{E}, g_{I}, t \mid v\right) \rho^{(v)}(v, t)$. Integration of Eq. (5.5) over $g_{E}, g_{I}$ yields

$$
\partial_{t} \rho_{\lambda}^{(v)}(v)=\partial_{v}\left\{\left[\left(\frac{v-\varepsilon_{r}}{\tau}\right)+\mu_{\lambda E}(v)\left(\frac{v-\varepsilon_{E}}{\tau}\right)+\mu_{\lambda I}(v)\left(\frac{v-\varepsilon_{I}}{\tau}\right)\right] \rho_{\lambda}^{(v)}(v)\right\} .
$$

Multiplying Eq. (5.5) with $g_{E}, g_{I}$, then integrating over $g_{E}, g_{I}$, respectively, upon using Eq. (5.14), leads to

$$
\begin{aligned}
\partial_{t} \mu_{\lambda E}(v) & =-\frac{1}{\sigma_{E}}\left[\mu_{\lambda E}(v)-\bar{g}_{\lambda E}(t)\right] \\
& +\left[\left(\frac{v-\varepsilon_{r}}{\tau}\right)+\mu_{\lambda E}(v)\left(\frac{v-\varepsilon_{E}}{\tau}\right)+\mu_{\lambda I}(v)\left(\frac{v-\varepsilon_{I}}{\tau}\right)\right]\left[\partial_{v} \mu_{\lambda E}(v)\right] \\
& +\left\{\frac{\Sigma_{\lambda E}^{2}(v)}{\rho_{\lambda}^{(v)}(v)} \partial_{v}\left[\left(\frac{v-\varepsilon_{E}}{\tau}\right) \rho_{\lambda}^{(v)}(v)\right]+\left[\partial_{v} \Sigma_{\lambda E}^{2}(v)\right]\left(\frac{v-\varepsilon_{E}}{\tau}\right)\right\} \\
& +\left\{\frac{C_{\lambda E I}(v)}{\rho_{\lambda}^{(v)}(v)} \partial_{v}\left[\left(\frac{v-\varepsilon_{I}}{\tau}\right) \rho_{\lambda}^{(v)}(v)\right]+\left[\partial_{v} C_{\lambda E I}(v)\right]\left(\frac{v-\varepsilon_{I}}{\tau}\right)\right\},
\end{aligned}
$$

and

$$
\begin{aligned}
\partial_{t} \mu_{\lambda I}(v) & =-\frac{1}{\sigma_{I}}\left[\mu_{\lambda I}(v)-\bar{g}_{\lambda I}(t)\right] \\
& +\left[\left(\frac{v-\varepsilon_{r}}{\tau}\right)+\mu_{\lambda E}(v)\left(\frac{v-\varepsilon_{E}}{\tau}\right)+\mu_{\lambda I}(v)\left(\frac{v-\varepsilon_{I}}{\tau}\right)\right]\left[\partial_{v} \mu_{\lambda I}(v)\right] \\
& +\left\{\frac{C_{\lambda E I}(v)}{\rho_{\lambda}^{(v)}(v)} \partial_{v}\left[\left(\frac{v-\varepsilon_{E}}{\tau}\right) \rho_{\lambda}^{(v)}(v)\right]+\left[\partial_{v} C_{\lambda E I}(v)\right]\left(\frac{v-\varepsilon_{E}}{\tau}\right)\right\} \\
& +\left\{\frac{\Sigma_{\lambda I}^{2}(v)}{\rho_{\lambda}^{(v)}(v)} \partial_{v}\left[\left(\frac{v-\varepsilon_{I}}{\tau}\right) \rho_{\lambda}^{(v)}(v)\right]+\left[\partial_{v} \Sigma_{\lambda I}^{2}(v)\right]\left(\frac{v-\varepsilon_{I}}{\tau}\right)\right\}
\end{aligned}
$$


where

$$
\begin{aligned}
\Sigma_{\lambda E}^{2}(v) & =\mu_{\lambda E}^{(2)}(v)-\mu_{\lambda E}^{2}(v), \quad \Sigma_{\lambda I}^{2}(v)=\mu_{\lambda I}^{(2)}(v)-\mu_{\lambda I}^{2}(v), \\
C_{\lambda E I}(v) & =\mu_{\lambda E I}^{(2)}(v)-\mu_{\lambda E}(v) \mu_{\lambda I}(v)
\end{aligned}
$$

are conditional variances and conditional correlation, respectively. Eqs. (5.14),(5.15), and (5.16) constitute the first equations in the infinite hierarchy of conditional expectation expansions.

\section{Closures:}

To truncate the infinite hierarchy of the equations governing conditional moments to a lower order to obtain essential effective dynamics, using the same intuition as described above, we postulate the following closure:

\section{Closure Condition I:}

$$
\Sigma_{\lambda E}^{2}(v)=\sigma_{\lambda E}^{2}(t), \quad \Sigma_{\lambda I}^{2}(v)=\sigma_{\lambda I}^{2}(t)
$$

which are similar to the closure used in obtaining the kinetic equations for a purely excitatory neuronal network.

\section{Closure Condition II:}

$$
C_{\lambda E I}(v)=0
$$

i.e., the conditional correlation of inhibitory conductance and the excitatory conductance vanishes (As shown above, if not conditioned on $v$, the inhibitory and excitatory conductances are uncorrelated).

With these closure conditions, naturally $\partial_{v} \Sigma_{\lambda E}^{2}(v)=0, \partial_{v} \Sigma_{\lambda I}^{2}(v)=0$, and we obtain the following equations for $\mu_{\lambda E}(v), \mu_{\lambda I}(v)$ and $\rho_{\lambda}^{(v)}(v)$ for $\lambda=E, I$ :

$$
\begin{aligned}
\partial_{t} \rho_{\lambda}^{(v)}(v)= & \partial_{v}\left\{\left[\left(\frac{v-\varepsilon_{r}}{\tau}\right)+\mu_{\lambda E}(v)\left(\frac{v-\varepsilon_{E}}{\tau}\right)+\mu_{\lambda I}(v)\left(\frac{v-\varepsilon_{I}}{\tau}\right)\right] \rho_{\lambda}^{(v)}(v)\right\} \\
\partial_{t} \mu_{\lambda E}(v)= & -\frac{1}{\sigma_{E}}\left[\mu_{\lambda E}(v)-\bar{g}_{\lambda E}(t)\right]+\frac{\sigma_{\lambda E}^{2}(t)}{\rho_{\lambda}^{(v)}(v)} \partial_{v}\left[\left(\frac{v-\varepsilon_{E}}{\tau}\right) \rho_{\lambda}^{(v)}(v)\right] \\
& +\left[\left(\frac{v-\varepsilon_{r}}{\tau}\right)+\mu_{\lambda E}(v)\left(\frac{v-\varepsilon_{E}}{\tau}\right)+\mu_{\lambda I}(v)\left(\frac{v-\varepsilon_{I}}{\tau}\right)\right]\left[\partial_{v} \mu_{\lambda E}(v)\right] \\
\partial_{t} \mu_{\lambda I}(v)= & -\frac{1}{\sigma_{I}}\left[\mu_{\lambda I}(v)-\bar{g}_{\lambda I}(t)\right]+\frac{\sigma_{\lambda I}^{2}(t)}{\rho_{\lambda}^{(v)}(v)} \partial_{v}\left[\left(\frac{v-\varepsilon_{I}}{\tau}\right) \rho_{\lambda}^{(v)}(v)\right] \\
& +\left[\left(\frac{v-\varepsilon_{r}}{\tau}\right)+\mu_{\lambda E}(v)\left(\frac{v-\varepsilon_{E}}{\tau}\right)+\mu_{\lambda I}(v)\left(\frac{v-\varepsilon_{I}}{\tau}\right)\right]\left[\partial_{v} \mu_{\lambda I}(v)\right]
\end{aligned}
$$

which now are closed with respect to $\mu_{\lambda E}(v), \mu_{\lambda I}(v)$ and $\rho_{\lambda}^{(v)}(v)$. Eqs (5.19) constitute our kinetic equations for dynamics of coupled excitatory and inhibitory networks. We note that, following the procedure in Sec. 4.3.4, boundary conditions can be similarly derived for Eqs. (5.19) by using Eqs. (5.3) and (5.4). Figure ( 5.1) illustrates the accuracy of this kinetic theory by comparing its solution with a full numerical simulation of I\&F networks with both excitatory and inhibitory neurons. 


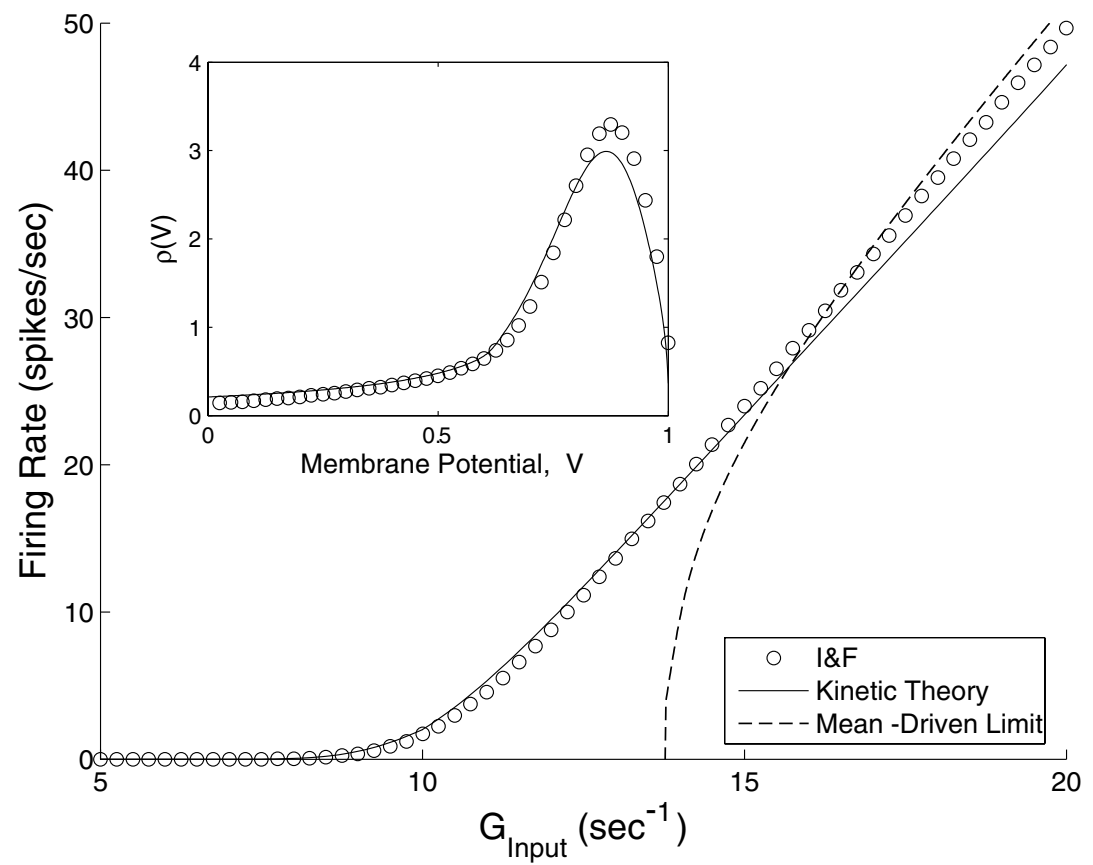

Fig. 5.1. Accuracy of Kinetic Theory for Neuronal Network Consisting of Both Excitatory and Inhibitory Neurons: The population-averaged firing rate per neuron $m$ for the excitatory population as a function of mean input conductance $G_{\text {input }}=f \nu_{0 E}$. Inset: probability density function of the membrane potential for the excitatory population. Circles: Simulation of the original I\&SF neuronal network dynamics (2.1) in a statistical steady state, under the shunting inhibition, i.e., $\varepsilon_{I}=\varepsilon_{r}$; Solid line: Kinetic Theory (4.19); Dashed line: Mean-Driven Limit (Eq. (4.11) extended to this network of both excitatory and inhibitory neurons). The coupling parameters are $S_{E E}=S_{I E}=0.1$, $S_{E I}=S_{I I}=0.5$, (Note that, for these particular couplings, either in the original IEG $F$ network (2.1) or in our kinetic theory (5.19), we have $m_{I}=m_{E}$, which can be easily concluded from the symmetries in these values of $S_{\lambda \lambda^{\prime}}, \lambda \lambda^{\prime}=E, I$, see Eqs. (2.1) and Eqs (5.6)). Other Parameters: $f=0.2 \mathrm{~ms}$, $\nu_{0 I}=0, \sigma_{E}=3 \mathrm{~ms}, \sigma_{I}=5 \mathrm{~ms}, \tau=20 \mathrm{~ms}, N_{E}=300, N_{I}=100$, and $p=0.25 . \quad\left(\nu_{0 E}=1300 \mathrm{~s}^{-1}\right.$ and $\nu_{0 I}=0$ for the inset.)

5.3. Fokker-Planck equations (The $\sigma_{E}, \sigma_{I} \rightarrow 0$ limit): Here, we point out that in order to obtain the Fokker-Planck equation similar to Eq. (4.24), there is no need to postulate Closure Condition II for the conditional correlation (Eq. (5.18)) even if the conditional correlation $C_{\lambda E I}(v)$ does not vanish in the dynamics of the network. By imposing only Closure Condition I, in the limit $\sigma_{E} \rightarrow 0, \sigma_{I} \rightarrow 0$, by noticing that $\sigma_{E} \sigma_{\lambda E}^{2} \sim \mathcal{O}(1)$ and $\sigma_{I} \sigma_{\lambda I}^{2} \sim \mathcal{O}(1)$, Eqs. (5.15) and (5.16) reduce to

$$
\begin{gathered}
\mu_{\lambda E}(v)=\bar{g}_{\lambda E}(t)+\frac{\sigma_{E} \sigma_{\lambda E}^{2}}{\rho_{\lambda}^{(v)}(v)} \partial_{v}\left[\left(\frac{v-\varepsilon_{E}}{\tau}\right) \rho_{\lambda}^{(v)}(v)\right], \\
\mu_{\lambda I}(v)=\bar{g}_{\lambda I}(t)+\frac{\sigma_{I} \sigma_{\lambda I}^{2}}{\rho_{\lambda}^{(v)}(v)} \partial_{v}\left[\left(\frac{v-\varepsilon_{I}}{\tau}\right) \rho_{\lambda}^{(v)}(v)\right] .
\end{gathered}
$$

Substituting these into Eq. (5.19a) results in Fokker-Planck equations for the dynamics of coupled excitatory and inhibitory neuronal networks:

$$
\partial_{t} \rho_{\lambda}^{(v)}(v)+\partial_{v} J_{\lambda}^{\mathrm{FP}}(v)=0
$$


$\lambda=E, I$, with the probability flux $J_{\lambda}^{\mathrm{FP}}(v)$ defined by

$$
\begin{aligned}
J_{\lambda}^{\mathrm{FP}}(v) \equiv & -\left[\left(\frac{v-\varepsilon_{r}}{\tau}\right)+\gamma_{\lambda E}(t)\left(\frac{v-\varepsilon_{E}}{\tau}\right)+\gamma_{\lambda I}(t)\left(\frac{v-\varepsilon_{I}}{\tau}\right)\right] \rho_{\lambda}^{(v)}(v) \\
& -\left[\sigma_{E} \sigma_{\lambda E}^{2}(t)\left(\frac{v-\varepsilon_{E}}{\tau}\right)^{2}+\sigma_{I} \sigma_{\lambda I}^{2}(t)\left(\frac{v-\varepsilon_{I}}{\tau}\right)^{2}\right] \partial_{v} \rho_{\lambda}^{(v)}(v) .
\end{aligned}
$$

where $\gamma_{\lambda E}(t) \equiv \bar{g}_{\lambda E}(t)+\sigma_{E} \sigma_{\lambda E}^{2}(t) / \tau, \gamma_{\lambda I}(t) \equiv \bar{g}_{\lambda I}(t)+\sigma_{I} \sigma_{\lambda I}^{2}(t) / \tau$.

6. Extension to many coarse-grained patches

In this article, we have presented a detailed statistical physics framework of capturing the effective dynamics of neuronal networks of both excitatory and inhibitory neurons. It is easy to extend our kinetic theory to include interactions among coarsegrained patches created by a spatial coarse-graining procedure. Let $\mathbf{x}$ be the coarsegrained coordinate labeling a patch, then $\rho_{\lambda}^{(v)}(v, t)$ and $\mu_{\lambda \lambda^{\prime}}(v, t)$, in Eqs (5.19), where $\lambda, \lambda^{\prime}=E, I$, are generalized to be $\rho_{\lambda}^{(v)}(\mathbf{x} ; v, t)$ and $\mu_{\lambda \lambda^{\prime}}(\mathbf{x} ; v, t)$ with the following coupling between coarse-grained patches:

$$
\begin{gathered}
\bar{g}_{\lambda E}(\mathbf{x} ; t)=f_{E}(\mathbf{x}) \nu_{0 E}(\mathbf{x} ; t)+\bar{S}_{\lambda E} \int K_{E}\left(\mathbf{x}, \mathbf{x}^{\prime}\right) m_{E}\left(\mathbf{x}^{\prime} ; t\right) d \mathbf{x}^{\prime}, \\
\bar{g}_{\lambda I}(\mathbf{x} ; t)=f_{I}(\mathbf{x}) \nu_{0 I}(\mathbf{x} ; t)+\bar{S}_{\lambda I} \int K_{I}\left(\mathbf{x}, \mathbf{x}^{\prime}\right) m_{I}\left(\mathbf{x}^{\prime} ; t\right) d \mathbf{x}^{\prime}, \\
\sigma_{\lambda E}^{2}(\mathbf{x} ; t)=\frac{1}{2 \sigma_{E}} f_{E}^{2}(\mathbf{x}) \nu_{0 E}(\mathbf{x} ; t)+\frac{\bar{S}_{\lambda E}^{2}}{2 \sigma_{E} p N_{E}(\mathbf{x})} \int K_{E}^{2}\left(\mathbf{x}, \mathbf{x}^{\prime}\right) m_{E}\left(\mathbf{x}^{\prime} ; t\right) d \mathbf{x}^{\prime}, \\
\sigma_{\lambda I}^{2}(\mathbf{x} ; t)=\frac{1}{2 \sigma_{I}} f_{I}^{2}(\mathbf{x}) \nu_{0 I}(\mathbf{x} ; t)+\frac{\bar{S}_{\lambda I}^{2}}{2 \sigma_{I} p N_{I}(\mathbf{x})} \int K_{I}^{2}\left(\mathbf{x}, \mathbf{x}^{\prime}\right) m_{I}\left(\mathbf{x}^{\prime} ; t\right) d \mathbf{x}^{\prime},
\end{gathered}
$$

which are the generalizations of Eqs. (5.6). Here, $K_{E, I}\left(\mathbf{x}, \mathbf{x}^{\prime}\right)$ are smoothed (i.e., coarse-grained) spatial interaction kernels for excitatory and inhibitory neurons, respectively.

Finally, we mention that our kinetic theory can be readily generalized to include a finite refractory period $\tau_{r e f}$, i.e, after reset, a neuron's voltage stays at $\varepsilon_{r}$ for a finite duration $\tau_{r e f}$, then follows the dynamics of Eq. (2.1a). This generalization will be published elsewhere.

Appendix A. The result (Eq. (3.3)) of a voltage jump upon receiving an input spike can also easily be seen as follows. The dynamics of integrate-and-fire neuron in response to a single input spike at time $t=0$ is described by

$$
\frac{d}{d t} V=-\frac{\left(V-\varepsilon_{r}\right)}{\tau}-\frac{f}{\tau} \delta(t)\left(V-\varepsilon_{E}\right),
$$

which can be interpreted as

$$
\frac{d V}{V-\varepsilon_{E}}=-\frac{1}{\tau}\left(\frac{V-\varepsilon_{r}}{V-\varepsilon_{E}}\right) d t-\frac{f}{\tau} \delta(t) d t .
$$

By integrating from $-\varepsilon$ to $+\varepsilon, \varepsilon \ll 1$, we have

$$
\ln \left(\frac{V\left(0^{+}\right)-\varepsilon_{E}}{V\left(0^{-}\right)-\varepsilon_{E}}\right)=O(\varepsilon)-\frac{f}{\tau} .
$$

Therefore, in the limit of $\varepsilon \rightarrow 0$, we obtain the jump (Eq. (3.3)) from Eq. (A.1). 
Appendix B. We repeat here the description of a network consisting of $N$ all excitatory neurons with an exponential time-course for conductances. The dynamics of an all-to-all coupled excitatory neuronal network are governed by

$$
\begin{aligned}
\tau \frac{d V_{i}}{d t} & =-\left(V_{i}-\varepsilon_{r}\right)-G_{i}(t)\left(V_{i}-\varepsilon_{E}\right) \\
\sigma \frac{d G_{i}}{d t} & =-G_{i}+f \sum_{\mu} \delta\left(t-t_{\mu}^{i}\right)+\frac{S}{N} \sum_{j} \sum_{\mu} p_{j \mu} \delta\left(t-t_{j \mu}\right)
\end{aligned}
$$

where $V_{i}$ is the membrane potential of the $i$ th neuron in the network and $\sigma$ is the decay time-scale of the excitatory conductance time-course. The external input is modeled by the second term in Eq. (B.1b), where $\left\{t_{\mu}^{i}\right\}$ is a Poisson spike train for the $i$ th neuron with rate $\nu_{0}(t)$. For each external input spike, the jump induced in conductance is $f / \sigma$. The third term in Eq. (B.1b) describes the spike input to the $i$ th neuron from the $j$ th neuron. $p_{j \mu}$ describes the probability of synaptic release, which is modelled by a Bernoulli process, with release probability equal to $p$, i.e., $p_{j \mu}=1$ with probability $p$; 0, otherwise. As discussed in Section 4, under certain conditions [39], it is reasonable to assume that the input spike train to the $i$ th neuron is Poisson with rate $p N m(t)$, where $m(t)$ is the population-averaged firing rate per neuron and $p N$ is an effective number of neurons that are coupled to neuron $i$. For each incoming spike from other neurons in the network, the total jump of the conductance is $\bar{S} /(p N \sigma)$, where $\bar{S}=p S$. We consider the probability density function:

$$
\rho(v, g, t)=\frac{1}{N} \sum_{i=1}^{N} \mathbb{E}\left[\delta\left(v-V_{i}(t)\right) \delta\left(g-G_{i}(t)\right)\right] .
$$

First, we evaluate $\rho(v, g, t+\Delta t)$. We use again the identity (Eq. (3.5)) to write

$$
\begin{aligned}
& \mathbb{E}\left[\delta\left(v-V_{i}(t+\Delta t)\right) \delta\left(g-G_{i}(t+\Delta t)\right)\right] \\
= & \mathbb{E}\left[\mathbb{E}\left[\delta\left(v-V_{i}(t+\Delta t)\right) \delta\left(g-G_{i}(t+\Delta t)\right) \mid V_{i}(t), G_{i}(t)\right]\right] .
\end{aligned}
$$

Given a neuron that has a voltage $V_{i}(t)$ and a conductance $G_{i}(t)$ at time $t$, with probability up to $\mathcal{O}(\Delta t)$, there will be three possibilities in the interval $(t, t+\Delta t)$ : (i) the neuron, with probability $\left(1-\nu_{0}(t) \Delta t\right)(1-p m(t) N \Delta t)$, receives no spikes from either external input nor other neurons in the network, and the neuron relaxes according to dynamics Eq. (B.1) without any $\delta$-functions on the right-hand side; (ii) the neuron, with probability $\nu_{0}(t) \Delta t(1-m(t) p N \Delta t)$, receives only one spike from the external input but no spikes from other neurons in the network, and its conductance jumps by the amount of $f / \sigma$; (iii) the neuron, with probability $p N m(t) \Delta t\left(1-\nu_{0}(t) \Delta t\right)$, receives only one spike from other neurons in the network but not from the external input, and its conductance jumps by the amount of $\bar{S} /(p N \sigma), \bar{S} \equiv p S$. Therefore, to $\mathcal{O}(\Delta t)$, we have

$$
\begin{aligned}
& \mathbb{E}\left[\delta\left(v-V_{i}(t+\Delta t)\right) \delta\left(g-G_{i}(t+\Delta t)\right) \mid V_{i}(t), G_{i}(t)\right] \\
= & \left(1-\nu_{0}(t) \Delta t\right)(1-p m(t) N \Delta t) \times \\
& \delta\left(v-\left[V_{i}(t)+\left[-\frac{1}{\tau}\left(V_{i}(t)-\varepsilon_{r}\right)-\frac{G_{i}(t)}{\tau}\left(V_{i}(t)-\varepsilon_{E}\right)\right] \Delta t\right]\right) \\
& \times \delta\left(g-\left[G_{i}(t)-\frac{1}{\sigma} G_{i}(t) \Delta t\right]\right)
\end{aligned}
$$




$$
\begin{aligned}
& +\nu_{0}(t) \Delta t \delta\left(v-V_{i}(t)\right) \delta\left(g-\left[G_{i}(t)+\frac{f}{\sigma}\right]\right) \\
& +p N m(t) \Delta t \delta\left(v-V_{i}(t)\right) \delta\left(g-\left[G_{i}(t)+\frac{\bar{S}}{p N \sigma}\right]\right) \\
& =\left(1-\nu_{0}(t) \Delta t\right)(1-p m(t) N \Delta t) \times \\
& \quad\left\{\delta\left(v-V_{i}(t)\right)+\frac{\partial}{\partial v} \delta\left(v-V_{i}(t)\right)\left[\frac{1}{\tau}\left(V_{i}(t)-\varepsilon_{r}\right)+\frac{G_{i}(t)}{\tau}\left(V_{i}(t)-\varepsilon_{E}\right)\right] \Delta t\right\} \\
& \times\left\{\delta\left(g-G_{i}(t)\right)+\frac{\partial}{\partial g} \delta\left(g-G_{i}(t)\right)\left[\frac{1}{\sigma} G_{i}(t) \Delta t\right]\right\} \\
& +\nu_{0}(t) \Delta t \delta\left(v-V_{i}(t)\right) \delta\left(g-\frac{f}{\sigma}-G_{i}(t)\right) \\
& +p N m(t) \Delta t \delta\left(v-V_{i}(t)\right) \delta\left(g-\frac{\bar{S}}{p N \sigma}-G_{i}(t)\right)
\end{aligned}
$$

with $\bar{S}=p S$. We can move terms, such as $\left[\frac{1}{\tau}\left(V_{i}(t)-\varepsilon_{r}\right)+\frac{G_{i}(t)}{\tau}\left(V_{i}(t)-\varepsilon_{E}\right)\right]$, inside the partial derivative $\partial / \partial v$. Thus

$$
\begin{aligned}
& \mathbb{E}\left[\delta\left(v-V_{i}(t+\Delta t)\right) \delta\left(g-G_{i}(t+\Delta t)\right) \mid V_{i}(t), G_{i}(t)\right] \\
= & {\left[1-\nu_{0}(t) \Delta t-p m(t) N \Delta t\right] \delta\left(v-V_{i}(t)\right) \delta\left(g-G_{i}(t)\right) } \\
& +\frac{\partial}{\partial v}\left[\delta\left(v-V_{i}(t)\right) \delta\left(g-G_{i}(t)\right)\left[\frac{1}{\tau}\left(V_{i}(t)-\varepsilon_{r}\right)+\frac{G_{i}(t)}{\tau}\left(V_{i}(t)-\varepsilon_{E}\right)\right]\right] \Delta t \\
& +\frac{\partial}{\partial g}\left[\delta\left(v-V_{i}(t)\right) \delta\left(g-G_{i}(t)\right) \frac{1}{\sigma} G_{i}(t) \Delta t\right] \\
& +\nu_{0}(t) \Delta t \delta\left(v-V_{i}(t)\right) \delta\left(g-\frac{f}{\sigma}-G_{i}(t)\right) \\
& +p N m(t) \Delta t \delta\left(v-V_{i}(t)\right) \delta\left(g-\frac{\bar{S}}{p N \sigma}-G_{i}(t)\right),
\end{aligned}
$$

which, after noticing $\delta(x-a) a=\delta(x-a) x$, yields

$$
\begin{aligned}
& \mathbb{E}\left[\delta\left(v-V_{i}(t+\Delta t)\right) \delta\left(g-G_{i}(t+\Delta t)\right) \mid V_{i}(t), G_{i}(t)\right] \\
= & \delta\left(v-V_{i}(t)\right) \delta\left(g-G_{i}(t)\right) \\
& +\frac{\partial}{\partial v}\left[\delta\left(v-V_{i}(t)\right) \delta\left(g-G_{i}(t)\right)\left[\frac{1}{\tau}\left(v-\varepsilon_{r}\right)+\frac{g}{\tau}\left(v-\varepsilon_{E}\right)\right]\right] \Delta t \\
& +\frac{\partial}{\partial g}\left[\delta\left(v-V_{i}(t)\right) \delta\left(g-G_{i}(t)\right) \frac{1}{\sigma} g \Delta t\right] \\
& +\nu_{0}(t) \Delta t\left[\delta\left(v-V_{i}(t)\right) \delta\left(g-\frac{f}{\sigma}-G_{i}(t)\right)-\delta\left(v-V_{i}(t)\right) \delta\left(g-G_{i}(t)\right)\right] \\
& +p N m(t) \Delta t\left[\delta\left(v-V_{i}(t)\right) \delta\left(g-\frac{\bar{S}}{p N \sigma}-G_{i}(t)\right)-\delta\left(v-V_{i}(t)\right) \delta\left(g-G_{i}(t)\right)\right] .
\end{aligned}
$$


By taking expectation over all possible trajectories of $\left\{V_{i}(t), G_{i}(t)\right\}$ and averaging over all neurons, we have

$$
\begin{aligned}
\partial_{t} \rho= & \partial_{v}\left[\left(\left(\frac{v-\varepsilon_{r}}{\tau}\right)+g\left(\frac{v-\varepsilon_{E}}{\tau}\right)\right) \rho\right]+\partial_{g}\left(\frac{g}{\sigma} \rho\right) \\
& +\nu_{0}(t)\left[\rho\left(v, g-\frac{f}{\sigma}, t\right)-\rho(v, g, t)\right]+p m(t) N\left[\rho\left(v, g-\frac{\bar{S}}{p N \sigma}, t\right)-\rho(v, g, t)\right]
\end{aligned}
$$

in the limit of $\Delta t \rightarrow 0$, where $\rho \equiv \rho(v, g, t)$. This is Eq. (4.2) in the main text.

Acknowledgement. The work was performed under the support of NSF Grant DMS-0506396 for D.C., L.T., and D.W.M., NSF Grant DMS-0211655 for D.W.M., D.C., and A.V.R., and a Sloan Fellowship for D.C.

\section{REFERENCES}

[1] D. Somers, S. Nelson and M. Sur, An emergent model of orientation selectivity in cat visual cortical simple cells, J. Neurosci., 15, 5448-5465, 1995.

[2] T. Troyer, A. Krukowski, N. Priebe and K. Miller, Contrast invariant orientation tuning in cat visual cortex with feedforward tuning and correlation based intracortical connectivity, J. Neurosci., 18, 5908-5927, 1998.

[3] D. McLaughlin, R. Shapley, M. Shelley and J. Wielaard, A neuronal network model of macaque primary visual cortex (V1): orientation selectivity and dynamics in the input layer $4 C \alpha$, Proc. Nat'l. Acad. Sci. USA, 97, 8087-8092, 2000.

[4] D. Cai, A. V. Rangan and D. W. McLaughlin, Architectural and synaptic mechanisms underlying coherent spontaneous activity in V1, Proc. Nat'l Acad. Sci. USA, 102, 5868-5873, 2005.

[5] A. V. Rangan, D. Cai and D. W. McLaughlin, Modeling the spatiotemporal cortical activity associated with the line-motion illusion in primary visual cortex, Proc. Nat'l Acad. Sci. USA, 102, 18793-18800, 2005

[6] T. Bonhoeffer and A. Grinvald, Iso-orientation domains in cat visual cortex are arranged in pinwheel like patterns, Nature, 353, 429-431, 1991.

[7] G. Blasdel, Differential imaging of ocular dominance and orientation selectivity in monkey striate cortex, J. Neurosci., 12, 3115-3138, 1992.

[8] G. Blasdel, Orientation selectivity, preference, and continuity in the monkey striate cortex, J. of Neurosci., 12, 3139-3161, 1992.

[9] R. Everson, A. Prashanth, M. Gabbay, B. Knight, L. Sirovich and E. Kaplan, Representation of spatial frequency and orientation in the visual cortex, Proc. Nat'l. Acad. Sci. USA, 95, 8334-8338, 1998.

[10] P. Maldonado, I. Godecke, C. Gray and T. Bonhoeffer, Orientation selectivity in pinwheel centers in cat striate cortex, Science, 276, 1551-1555, 1997.

[11] J. Anderson, I. Lampl, D. Gillespie and D. Ferster, The contribution of noise to contrast invariance of orientation tuning in cat visual cortex, Science, 290, 1968-1972, 2000.

[12] E. A. Stern, A. E. Kincaid and C. J. Wilson, Spontaneous subthreshold membrane potential fluctuations and action potential variability of rat corticostriatal and striatal neurons in vivo, J. Neurophysiol., 77, 1697-1715, 1997.

[13] M. Shelley and D. McLaughlin, Coarse-grained reduction and analysis of a network model of cortical response. I. drifting grating stimuli, J. Comp. Neurosci., 12, 97-122, 2002.

[14] N. Fourcaud and N. Brunel, Dynamics of the firing probability of noisy integrate-and-fire neurons, Neural Comp., 14, 2057-2110, 2002.

[15] C. van Vreeswijk and H. Sompolinsky, Chaos in neuronal networks with balanced excitatory and inhibitory activity, Science, 274, 1724-1726, 1996.

[16] C. van Vreeswijk and H. Sompolinsky, Chaotic balanced state in a model of cortical circuits, Neural Comput., 15, 1321-1371, 1998.

[17] D. Cai, L. Tao, M. Shelley and D. W. Mclaughlin, An effective representation of fluctuationdriven neuronal networks with application to simple and complex cells in visual cortex, Proc. Nat'l. Acad. Sci. USA, 101, 7757-7762, 2004

[18] L. Tao, D. Cai, D. W. Mclaughlin, M. Shelley and R. Shapley, Orientation selectivity in primary visual cortex by fluctuation-controlled criticality, Proc. Nat'l. Acad. Sci. USA, submitted, 2006. 
[19] D. Cai, L. Tao and D. W. McLaughlin, An embedded network approach for scale-up of fluctuation-driven systems with preservation of spike information, Proc. Nat'l. Acad. Sci. USA, 101, 14288-14293, 2004.

[20] B. Knight, Dynamics of encoding in a populaton neurons, J. Gen. Physiol., 59, 734-766, 1972.

[21] W. J. Wilbur and J. Rinzel, A theoretical basis for large coefficient of variation and bimodality in neuronal interspike interval distributions, J. Theor. Biol, 105, 345-368, 1983.

[22] L. F. Abbott and C. van Vreeswijk, Asynchronous states in networks of pulse-coupled oscillators, Phys. Rev. E, 48, 1483-1490, 1993.

[23] A. Treves, Mean field analysis of neuronal spike dynamics, Network, 4, 259-284, 1993.

[24] T. Chawanya, A. Aoyagi, T. Nishikawa, K. Okuda and Y. Kuramoto, A model for feature linking via collective oscillations in the primary visual cortex, Biol. Cybern., 68, 483-90, 1993.

[25] G. Barna, T. Grobler and P. Erdi, Statistical model of the hippocampal CA3 region, II. the population framework: model of rhythmic activity in CA3 slice, Biol. Cybern., 79, 309321, 1998.

[26] J. Pham, K. Pakdaman, J. Champagnat and J. Vibert, Activity in sparsely connected excitatory neural networks: effect of connectivity neural networks, Neural Networks, 11, 415-434, 1998.

[27] N. Brunel and V. Hakim, Fast global oscillations in networks of integrate-and-fire neurons with low firing rates, Neural Comp., 11, 1621-1671, 1999.

[28] W. Gerstner, Population dynamics of spiking neurons: fast transients, asynchronous states, and locking, Neural Comp., 12, 43-80, 2000.

[29] A. Omurtag, B. W. Knight and L. Sirovich, On the simulation of large populations of neurons, J. Comp. Neurosci., 8, 51-63, 2000.

[30] A. Omurtag, E. Kaplan, B. W. Knight and L. Sirovich, A population approach to cortical dynamics with an application to orientation tuning, Network: Comput. Neural Syst., 11, 247-260, 2000.

[31] D. Nykamp and D. Tranchina, A population density method that facilitates large-scale modeling of neural networks: analysis and application to orientation tuning, J. Comp. Neurosci., 8, 19-50, 2000.

[32] D. Nykamp and D. Tranchina, A population density method that facilitates large-scale modeling of neural networks: extension to slow inhibitory synapses, Neural Comput., 13, 511-546, 2001.

[33] E. Haskell, D. Q. Nykamp and D. Tranchina, Population density methods for large-scale modeling of neuronal networks with realistic synaptic kinetics: cutting the dimension down to size, Network: Compt. Neural. Syst, 12, 141-174, 2001.

[34] H. Wilson and J. Cowan, A mathematical theory of the functional dynamics of cortical and thalamic nervous tissue, Kybernetik, 13, 55-80, 1973.

[35] B. Knight, D. Manin and L. Sirovich, Dynamical models of interacting neuron populations, In E. Gerf, editor, Symposium on Robotics and Cybernetics: Computational Engineering in Systems Applications. Cite Scientifique, Lille, France, 1996.

[36] A. Casti, A. Omurtag, A. Sornborger, E. Kaplan, B. Knight, J. Victor and L. Sirovich, A population study of integrate-and-fire-or-burst neurons, Neural Computation, 14, 957-986, 2002.

[37] A. V. Rangan, D. Cai and L.Tao, Numerical methods for solving kinetic equations of neuronal network dynamics, preprint, 2005.

[38] C. Koch, Biophysics of Computation, Oxford University Press, Oxford, 1999.

[39] E. Cinlar, Superposition of point processes, In P. A. W. Lewis, editor, Stochastic Point Processes: Statistical Analysis, Theory, and Applications, Wiley, New York, NY, 549-606, 1972 . 